\title{
Approximation by periodic multivariate quasi-projection operators *
}

\author{
Yu. Kolomoitsev ${ }^{1,2}$, A. Krivoshein ${ }^{3}$ and M. Skopina ${ }^{3}$ \\ ${ }^{1}$ Universität zu Lübeck, Institut für Mathematik, Lübeck, Germany \\ ${ }^{2}$ Institute of Applied Mathematics and Mechanics of NAS of Ukraine, Slov'yans'k, Ukraine \\ ${ }^{3}$ St. Petersburg State University, Russia \\ kolomoitsev@math.uni-luebeck.de, a.krivoshein@spbu.ru, skopina@ms1167.spb.edu
}

\begin{abstract}
Approximation properties of periodic quasi-projection operators with matrix dilations are studied. Such operators are generated by a sequence of functions $\varphi_{j}$ and a sequence of distributions/functions $\widetilde{\varphi}_{j}$. Error estimates for sampling-type quasi-projection operators are obtained under the periodic Strang-Fix conditions for $\varphi_{j}$ and the compatibility conditions for $\varphi_{j}$ and $\widetilde{\varphi}_{j}$. These estimates are given in terms of the Fourier coefficients of approximated functions and provide analogs of some known non-periodic results. Under some additional assumptions error estimates are given in other terms in particular using the best approximation. A number of examples are provided.
\end{abstract}

Keywords. Periodic quasi-projection operators, Sampling-type operators, Kantorovich-type operators, Periodic Strang-Fix conditions, Matrix dilation, Error of approximation, Best approximation, Wiener's classes.

AMS Subject Classification. 42B05, 42A10, 94A20

\section{Introduction}

Approximation properties of non-periodic quasi-projection operators are actively studied by many authors. The class of such operators is very large, it includes classical sampling and sampling-type expansions (see, e.g., 40, 42, 6, 37, 1, 9, 8, 17, 22, 24, 26] and the references therein), KantorovichKotelnikov operators and their generalizations (see, e.g., 2, 30, 10, 12, 11, 41, 25]), scaling expansions associated with wavelet constructions (see, e.g., 19, 20, 4, 5, 18, 23, 38) and others. The most general form of the multivariate quasi-projection operator with a dilation matrix $M$ is given by

$$
\sum_{k \in \mathbb{Z}^{d}} m^{j}\left\langle f, \widetilde{\varphi}\left(M^{j} \cdot-k\right)\right\rangle \varphi\left(M^{j} \cdot-k\right),
$$

where $\varphi$ is a function and $\widetilde{\varphi}$ is a distribution or function, $m=|\operatorname{det} M|$, and the inner product $\left\langle f, \widetilde{\varphi}\left(M^{j} \cdot-k\right)\right\rangle$ has meaning in some sense.

For a suitable function/distribution $\varphi$, the periodization of $m^{j} \varphi\left(M^{j} \cdot\right)$ leads to the sequence $\left\{\varphi_{j}\right\}_{j}$ of periodic functions/distributions such that the $k$-th Fourier coefficient of $\varphi_{j}$ is equal to $\widehat{\varphi}\left(M^{*-j} k\right)$,

\footnotetext{
${ }^{*}$ The first author was partially supported by DFG project KO 5804/1-1 (Section 4.2 belongs to this author); the second and the third authors are supported by the Russian Science Foundation under grant No. 18-11-00055 (Sections 4.1 and 5 belong to these authors)
} 
where $\widehat{\varphi}$ is the Fourier transform of $\varphi$. So, in the periodic case, the quasi-projection operators take the form

$$
Q_{j}\left(f, \varphi_{j}, \widetilde{\varphi}_{j}\right)=\frac{1}{m^{j}} \sum_{k}\left\langle f, \widetilde{\varphi}_{j}\left(\cdot-M^{-j} k\right)\right\rangle \varphi_{j}\left(\cdot-M^{-j} k\right),
$$

where the sum over $k$ is finite due to the periodicity of $\varphi_{j}$ and the multiplier $\frac{1}{m^{j}}$ is for a suitable normalization (see Section 3 for more details). In particular, the periodization of the classical sampling expansion, where $\widetilde{\varphi}$ is the Dirac delta-function and $\varphi$ is the sinc-function, leads to $Q_{j}\left(f, \varphi_{j}, \widetilde{\varphi}_{j}\right)$, where $\widetilde{\varphi}_{j}$ is a periodic distribution whose Fourier coefficients are $\widehat{\widetilde{\varphi}_{j}}(k)=1, k \in \mathbb{Z}^{d}$, and $\varphi_{j}=m^{j} \Lambda_{j}$, where $\Lambda_{j}$ is a 1 -periodic fundamental interpolant on a grid $M^{-j} k$, i.e. $\Lambda_{j}\left(M^{-j} k\right)=\delta_{0 k}$, $k \in M^{j}[-1 / 2,1 / 2)^{d} \cap \mathbb{Z}^{d}$. It is easy to see that in this case, $Q_{j}\left(f, \varphi_{j}, \widetilde{\varphi}_{j}\right)$ takes a sampling form

$$
Q_{j}\left(f, \varphi_{j}, \widetilde{\varphi}_{j}\right)=P_{j}(f)=\sum_{k} f\left(M^{-j} k\right) \Lambda_{j}\left(\cdot-M^{-j} k\right) .
$$

Univariate and multivariate fundamental interpolants $\Lambda_{j}$ on the uniform grids and the corresponding quasi-projection operators $P_{j}(f)$ were investigated by many authors (see, e.g., [28, 13, 36, 31, 32, 35]).

It is well known that in the non-periodic case some compatibility of $\varphi$ and $\widetilde{\varphi}$ and the Strang-Fix conditions for $\varphi$ are required for successful error estimates. The periodic Strang-Fix conditions were introduced in [7. For the case of the diagonal dilation matrix $M=2 I_{d}$ under the periodic StrangFix conditions of order $s>d / 2$ for the sequence $\left\{\Lambda_{j}\right\}$, the results in [7] and [34] yield the following error estimate for $P_{j}$ in $L_{2}$ norm

$$
\left\|f-P_{j}(f)\right\|_{2} \leq C 2^{-j \min \{\gamma, s\}}\|f\|_{H_{2}^{\max \{\gamma, s\}}},
$$

where $\gamma>d / 2$ and $H_{2}^{r}$ is the Sobolev space of order $r$.

Many results in harmonic analysis involve spaces described in terms of the Fourier transform (in the non-periodic case) such as the Fourier algebra and different its generalizations, see, e.g., [15, 27. Similarly, in the periodic case, spaces described in terms of the Fourier coefficients appear. In particular, a natural class of such spaces, including the Sobolev space $H_{2}^{r}$ and the Wiener algebra, consists of the spaces $A_{q}^{\alpha}$ such that the $A_{q}^{\alpha}$-norm is a weighted $\ell_{q}$-norm of the sequence of Fourier coefficients (see Section 2). For the case $M=2 I_{d}$, under the strengthened Strang-Fix conditions of order $s$ the following error estimate for $P_{j}$ in the $A_{q}^{\alpha}$-norm was obtained by Sprengel 35 .

$$
\left\|f-P_{j}(f)\right\|_{A_{q}^{\alpha}} \leq C 2^{-j \min \{\gamma-\alpha, s\}}\|f\|_{A_{q}^{\gamma}},
$$

where $q \geq 1, \gamma \geq \alpha \geq 0$, and $\gamma>d(1-1 / q)$. For lattices generated by a matrix $M$ whose eigenvalues are greater or equal (in absolute value) than 2 , an analogous estimate was obtained by Bergmann and Prestin in [3].

The goal of this paper is to obtain periodic analogs of some author's results in [22, 24, 25]. Namely, in 22] the error analysis of non-periodic quasi-projection operators (11) was given for a class of tempered distributions $\widetilde{\varphi}$, including the Dirac delta-function, and for a wide class of functions $\varphi$ with enough decay of $\varphi$ itself as well as its Fourier transform $\widehat{\varphi}$. Error estimates in $L_{p}$-norm, $p \geq 2$, were obtained under the assumptions of the Strang-Fix conditions of order $s$ for $\varphi$ and the weak compatibility of $\varphi$ and $\widetilde{\varphi}$ of order $s$ (which means vanishing of all derivatives up to order $s$ in the origin of the function $1-\widehat{\widehat{\varphi}} \bar{\varphi}$ ). The obtained estimates show that the approximation order depends on the smoothness of $f$ and on $s$. In particular, it was established that the approximation order equals $s$ for smooth enough functions $f$. Similar results were obtained in [24] for the same class of distributions $\widetilde{\varphi}$ and a class of band-limited functions $\varphi$, including the sinc-function, under the assumption of strict compatibility of $\varphi$ and $\widetilde{\varphi}$ (which means that the function $1-\widehat{\widetilde{\varphi}} \bar{\varphi}$ is identical 
zero in a neighborhood of the origin). In this case, it was shown that the approximation order depends only on the smoothness of $f$. In [25], Kantorovich-Kotelnikov type operators, that are the quasi-projection operators (1) with summable $\widetilde{\varphi}$ and band-limited $\varphi$, were investigated. These operators are bounded in $L_{p}$ for $1<p<\infty$. Under the assumption of weak compatibility of $\varphi$ and $\widetilde{\varphi}$ of order $s$, the $L_{p}$-rate of convergence was given in terms of the classical moduli of smoothness of order $s$.

The paper is organized as follows. Section 2 is devoted to notation and basic definitions. A wide class of periodic sampling-type quasi-projection operators $Q_{j}\left(f, \varphi_{j}, \widetilde{\varphi}_{j}\right)$ with matrix dilations is introduced in Section 3. The main results are presented in Section 4. Error estimates in terms of the Fourier coefficients of the approximated function is given in Section 4.1. Under the Strang-Fix conditions of order $s$ for $\varphi_{j}$ and the weak compatibility of order $s$ of functions $\varphi_{j}$ and distributions $\widetilde{\varphi}_{j}$, an error estimate in the $A_{q}^{\alpha}$-norm is obtained in Theorem [ 6 . Under the same assumptions, an error estimate for $Q_{j}$ in the $L_{p}$-norm, $p \geq 2$, is obtained in Theorem 9 . This theorem provides a periodic analog of the results obtained in [22. In Section 4.2, under some additional assumptions on the distributions $\widetilde{\varphi}_{j}$ and the matrix dilations $M$, we give several improvements of the error estimates obtained in the previous section. Particulary, if $\varphi_{j}$ and $\widetilde{\varphi}_{j}$ are strongly compatible, then we show that the error estimate for $Q_{j}\left(f, \varphi_{j}, \widetilde{\varphi}_{j}\right)$ can be given only in terms of the best approximation (see Theorem 19), but if $\varphi_{j}$ and $\widetilde{\varphi}_{j}$ are weakly compatible, then the corresponding estimates are given simultaneously in terms of Fourier coefficients and the best approximation (see Theorem 22). The case of Kantorovich-type quasi-projection operators (i.e., $\widetilde{\varphi}_{j}$ is an integrable function) is also considered. In Section 5, we provide some examples.

\section{Notation}

We use the standard multi-index notations. Let $\mathbb{N}$ be the set of positive integers, $\mathbb{R}^{d}$ be the $d$ dimensional Euclidean space, $\mathbb{Z}^{d}$ be the integer lattice in $\mathbb{R}^{d}, \mathbb{T}^{d}=\mathbb{R}^{d} / \mathbb{Z}^{d}$ be the $d$-dimensional torus. Let $x=\left(x_{1}, \ldots, x_{d}\right)^{T}$ and $y=\left(y_{1}, \ldots, y_{d}\right)^{T}$ be column vectors in $\mathbb{R}^{d}$, then $(x, y):=x_{1} y_{1}+\cdots+x_{d} y_{d}$, $|x|:=\sqrt{(x, x)} ; \mathbf{0}=(0, \ldots, 0)^{T} \in \mathbb{R}^{d} ; \mathbb{Z}_{+}^{d}:=\left\{x \in \mathbb{Z}^{d}: x_{k} \geq 0, k=1, \ldots, d\right\}$. If $a \in \mathbb{R}^{d}, r>0$, then $B_{r}(a)$ denotes the ball of radius $r$ with the center in $a$.

$$
\text { If } \alpha \in \mathbb{Z}_{+}^{d} \text {, we set }[\alpha]=\sum_{k=1}^{d} \alpha_{k}, D^{\alpha} f=\frac{\partial^{[\alpha]} f}{\partial^{\alpha} x_{1} \ldots \partial^{\alpha} d x_{d}} \text {. }
$$

If $A$ is a $d \times d$ matrix, then $\|A\|$ denotes its operator norm in $\mathbb{R}^{d} ; A^{*}$ denotes the conjugate matrix to $A$.

Let $M$ be a dilation matrix, i.e. an integer valued $d \times d$ matrix, such that the absolute value of each its eigenvalue is greater than $1, m:=|\operatorname{det} M|, D(M):=M[-1 / 2,1 / 2)^{d} \cap \mathbb{Z}^{d}$. It is known (see, e.g., [21, Chapter 2]) that $D(M)$ is a set of digits of $M$, and any $k \in \mathbb{Z}^{d}$ can be uniquely represented as $k=M n+r, r \in D(M), n \in \mathbb{Z}^{d}$.

Since the spectrum of the operator $M^{-1}$ is located in $B_{r}(\mathbf{0})$, where $r=r\left(M^{-1}\right):=\lim _{j \rightarrow+\infty}\left\|M^{-j}\right\|^{\frac{1}{j}}$ is the spectral radius of $M^{-1}$, and there exists at least one point of the spectrum on the boundary of the ball, we have

$$
\left\|M^{-j}\right\| \leq C_{M, \vartheta} \vartheta^{-j}, \quad j \geq 0,
$$

for every positive number $\vartheta$ whose absolute value is smaller than absolute value of any eigenvalue of $M$. In particular, we can take $\vartheta>1$ and, hence, $\lim _{j \rightarrow+\infty}\left\|M^{-j}\right\|=0$.

A matrix $M$ is called isotropic if it is similar to a diagonal matrix such that numbers $\lambda_{1}, \ldots, \lambda_{d}$ are placed on the main diagonal and $\left|\lambda_{1}\right|=\cdots=\left|\lambda_{d}\right|$. Thus, $\lambda_{1}, \ldots, \lambda_{d}$ are eigenvalues of $M$ and the spectral radius of $M$ is equal to $|\lambda|$, where $\lambda$ is one of the eigenvalues of $M$. Note that if the matrix $M$ is isotropic then $M^{*}$ and $M^{j}$ are isotropic for all $j \in \mathbb{Z}$. It is well known that for an isotropic matrix $M$ and for any $j \in \mathbb{Z}$ we have

$$
C_{1}^{M}|\lambda|^{j} \leq\left\|M^{j}\right\| \leq C_{2}^{M}|\lambda|^{j}, \quad j \in \mathbb{Z}
$$


where $\lambda$ is one of the eigenvalues of $M$, constants $C_{1}^{M}, C_{2}^{M}$ do not depend on $j$.

We will use notation $L_{p}$ for the space $L_{p}\left(\mathbb{T}^{d}\right)$ with the usual norm $\|f\|_{p}=\left(\int_{\mathbb{T}^{d}}|f(x)|^{p} d x\right)^{1 / p}$ for $1 \leq p<\infty$, and $\|f\|_{\infty}=$ vraisup $|f|$.

If $f \in L_{1}$, then $\widehat{f}(k), k \in \mathbb{Z}^{d}$, denotes the $k$-th Fourier coefficient of $f$.

For $g \in L_{1}$, we will use the following notation $g^{-}(t)=\overline{g(-t)}$.

As usual, the convolution of appropriate functions $f$ and $g$ is given by

$$
(f * g)(x)=\int_{\mathbb{T}^{d}} f(x-t) g(t) d t .
$$

We will use notation $\theta_{\alpha}(x):=\left(1+|x|^{2}\right)^{\frac{\alpha}{2}}, \alpha \geq 0, x \in \mathbb{R}^{d}$. It is easy to check that

$$
\theta_{\alpha}(x+y) \leq \theta_{\alpha}(x) \theta_{\alpha}(y) \quad \forall x, y \in \mathbb{R}^{d} .
$$

Since $\|M\|>1$ for any dilation matrix $M$, and hence $\left\|M^{* j}\right\|>1$ for all $j \in \mathbb{N}$, we have

$$
\theta_{\alpha}(x)=\theta_{\alpha}\left(M^{* j} M^{*-j} x\right) \leq\left\|M^{* j}\right\|^{\alpha} \theta_{\alpha}\left(M^{*-j} x\right) .
$$

Also, for $\alpha \geq 0$ and $1 \leq q \leq \infty$, we will use notation

$$
\ell_{q}^{\alpha}:=\left\{x=\left\{x_{k}\right\}_{k}:\left\{\theta_{\alpha}(k) x_{k}\right\}_{k} \in \ell_{q}\right\}, \quad \ell_{q}=\ell_{q}\left(\mathbb{Z}^{d}\right),
$$

and $\|x\|_{\ell_{q}^{\alpha}}=\left\|\left\{\theta_{\alpha}(k) x_{k}\right\}_{k}\right\|_{\ell_{q}}$ for $x \in \ell_{q}^{\alpha}$.

Let $1 \leq q \leq \infty$ and $\alpha \geq 0$. The space $A_{q}^{\alpha}$ of periodic functions is defined by

$$
A_{q}^{\alpha}:=\left\{f \in L_{1}:\{\widehat{f}(k)\}_{k} \in \ell_{q}^{\alpha}\right\}, \quad\|f\|_{A_{q}^{\alpha}}:=\left\|\{\widehat{f}(k)\}_{k}\right\|_{\ell_{q}^{\alpha}} .
$$

These spaces include the Wiener algebra $A:=A_{1}^{0}$ of functions with absolutely convergent Fourier series. It is clear that $A_{q}^{\beta} \subset A_{q}^{\alpha}$ for $\beta \geq \alpha$. If $q=2$, then the space $A_{2}^{\alpha}$ coincides with the Sobolev space $H_{2}^{\alpha}$. So $\alpha$ can be considered as a smoothness parameter. However, these smoothness properties (except $p=2$ ) differ from usual smoothness of the fractional Sobolev spaces $H_{p}^{\alpha}$. Some embeddings of $A_{q}^{\alpha}$ into $H_{p}^{\beta}$ and vice versa can be found in [35.

For a function $f \in A_{q}^{\alpha}$, we set

$$
\|f\|_{A_{q}^{\alpha}, j}^{O u t}:=\left(\sum_{k \notin D\left(M^{* j}\right)}|\widehat{f}(k)|^{q} \theta_{\alpha}^{q}(k)\right)^{1 / q}, \quad\|f\|_{A_{q}^{\alpha}, j}^{I n}:=\left(\sum_{k \in D\left(M^{* j}\right)}|\widehat{f}(k)|^{q} \theta_{\alpha}^{q}(k)\right)^{1 / q} .
$$

for $1 \leq q<\infty$, and

$$
\|f\|_{A_{\infty}^{\alpha}, j}^{O u t}:=\sup _{k \notin D\left(M^{* j}\right)}|\widehat{f}(k)| \theta_{\alpha}(k), \quad\|f\|_{A_{\infty}^{\alpha}, j}^{I n}:=\sup _{k \in D\left(M^{* j}\right)}|\widehat{f}(k)| \theta_{\alpha}(k)
$$

for $q=\infty$. For convenience, we will also use the following notation:

$$
\|f\|_{A_{q}^{\alpha}, j}^{q, \text { Out }}:=\sum_{k \notin D\left(M^{* j}\right)}|\widehat{f}(k)|^{q} \theta_{\alpha}^{q}(k), \quad\|f\|_{A_{q}^{\alpha}, j}^{q, I n}:=\sum_{k \in D\left(M^{* j}\right)}|\widehat{f}(k)|^{q} \theta_{\alpha}^{q}(k) .
$$

Let $\mathcal{D}=C^{\infty}\left(\mathbb{T}^{d}\right)$ be the space of infinitely differentiable functions on $\mathbb{R}^{d}$ that are periodic with period 1. A continuous linear functional on the space $\mathcal{D}$ is a periodic distribution. The linear space of periodic distributions we denote by $\mathcal{D}^{\prime}$. For a periodic distribution $\varphi \in \mathcal{D}^{\prime}$ and a function $f \in \mathcal{D}$, we denote the action of $\varphi$ on $f$ by $\varphi(f)$. For convenience, we will use notation $\langle\varphi, f\rangle:=\varphi(\bar{f})$ and 
$\langle f, \varphi\rangle=\overline{\langle\varphi, f\rangle}$. It is known (see, e.g., [14, p. 322], [33, p. 144]) that any periodic distribution $\varphi$ can be expanded in a weakly convergent (in $\mathcal{D}^{\prime}$ ) Fourier series

$$
\varphi(x)=\sum_{n \in \mathbb{Z}^{d}} \widehat{\varphi}(n) e^{2 \pi i(n, x)},
$$

where the sequence $\{\widehat{\varphi}(n)\}_{n}$ has at most polynomial growth and for any $f \in \mathcal{D}$

$$
\langle f, \varphi\rangle=\sum_{n \in \mathbb{Z}^{d}} \widehat{f}(n) \overline{\hat{\varphi}(n)} .
$$

Also, conversely, for any sequence $\{\widehat{\varphi}(n)\}_{n}$ of at most polynomial growth the series in the right-hand side of (7) converges weakly to a periodic distribution. The numbers $\widehat{\varphi}(n)$ are called the Fourier coefficients of a periodic distribution $\varphi$ and $\widehat{\varphi}(n)=\left\langle\varphi, e^{2 \pi i(n, \cdot)}\right\rangle=\varphi\left(e^{-2 \pi i(n, \cdot)}\right)$. The convolution of $f \in \mathcal{D}$ and the distribution $\varphi$ is defined by $(f * \varphi)(x)=\langle f, \overline{\varphi(x-\cdot)}\rangle$.

\section{Sampling-type quasi-projection operators}

In this section, we define the periodic quasi-projection operators $Q_{j}\left(f, \varphi_{j}, \widetilde{\varphi}_{j}\right)$, where $\varphi \in L_{1}, \widetilde{\varphi}_{j} \in$ $\mathcal{D}^{\prime}, j \in \mathbb{N}$, by

$$
Q_{j}\left(f, \varphi_{j}, \widetilde{\varphi}_{j}\right)=\frac{1}{m^{j}} \sum_{k \in D\left(M^{j}\right)}\left\langle f, \widetilde{\varphi}_{j}\left(\cdot-M^{-j} k\right)\right\rangle \varphi_{j}\left(\cdot-M^{-j} k\right) .
$$

By the definition of $\mathcal{D}^{\prime}$, such an operator $Q_{j}$ has meaning only for $f \in \mathcal{D}$, and

$$
\left\langle f, \widetilde{\varphi}_{j}\left(\cdot-M^{-j} k\right)\right\rangle=\sum_{l \in \mathbb{Z}^{d}} \widehat{f}(l) \overline{\widehat{\varphi}_{j}(l)} e^{2 \pi i\left(k, M^{*-j} l\right)} .
$$

But since the Fourier coefficients of $\widetilde{\varphi}_{j}$ have polynomial growth, the latter series converges for any $f$ whose Fourier coefficients decay sufficiently fast. Thus, to extend the class of functions $f$, we define the inner product $\left\langle f, \widetilde{\varphi}_{j}\left(\cdot-M^{-j} k\right)\right\rangle$ by (8) .

Next, we introduce several conditions on the sequences of functions $\varphi_{j}$ and distributions $\widetilde{\varphi}_{j}$ under which the quasi-projection operator $Q_{j}\left(f, \varphi_{j}, \widetilde{\varphi}_{j}\right)$ provides good enough approximation of an appropriate function $f$. For additional motivation of these conditions, we consider their connection with the analogous conditions for the non-periodic case (see [22, 24]). For this, we note that the periodic and non-periodic cases can be connected via periodization as follows. Let $\varphi \in L_{1}\left(\mathbb{R}^{d}\right)$ with $\{\widehat{\varphi}(k)\}_{k \in \mathbb{Z}^{d}} \in \ell_{1}$. Setting $\varphi_{j, l}(x):=m^{j / 2} \varphi\left(M^{j} x+l\right)$ and

$$
\varphi_{j}(x):=m^{j} \sum_{k} \varphi\left(M^{j}(x+k)\right)=m^{j / 2} \sum_{k} \varphi_{j, 0}(x+k),
$$

where the multiplier $m^{j / 2}$ is for a suitable normalization, we have that each $\varphi_{j}$ is in $L_{1}$ and by the Poisson summation formula,

$$
\varphi_{j}(x)=m^{j / 2} \sum_{k} \widehat{\varphi_{j, 0}}(k) e^{2 \pi i(k, x)}=\sum_{k} \widehat{\varphi}\left(M^{*-j} k\right) e^{2 \pi i(k, x)}=\sum_{k} \widehat{\varphi_{j}}(k) e^{2 \pi i(k, x)},
$$

which implies that $\widehat{\varphi_{j}}(k)=\widehat{\varphi}\left(M^{*-j} k\right)$.

1. The conditions on the growth of order $N \geq 0$ for Fourier coefficients of $\left\{\widetilde{\varphi}_{j}\right\}_{j}$ :

$$
\begin{array}{rlrl} 
& \left|\widehat{\widetilde{\varphi}_{j}}(k)\right| \leq C_{\widetilde{\varphi}}\left|M^{*-j} k\right|^{N}, & & \forall k \notin D\left(M^{* j}\right), \quad \forall j \in \mathbb{N}, \\
\max _{k \in D\left(M^{* j}\right)}\left|\widehat{\widetilde{\varphi}_{j}}(k)\right| \leq C_{\widetilde{\varphi}}, & & \forall j \in \mathbb{N} .
\end{array}
$$


These conditions correspond to the following non-periodic conditions:

$$
|\widehat{\widetilde{\varphi}}(\xi)| \leq C_{\widetilde{\varphi}}|\xi|^{N} \quad \text { for } \quad \xi \notin[-1 / 2,1 / 2]^{d}
$$

and

$$
|\widehat{\widetilde{\varphi}}(\xi)| \leq C_{\widetilde{\varphi}} \quad \text { for } \quad \xi \in[-1 / 2,1 / 2]^{d} .
$$

To show this, we note that $\widehat{\widetilde{\varphi}_{j}}(k)=\widehat{\widetilde{\varphi}}\left(M^{*-j} k\right)$. Thus, if non-periodic conditions are valid for $\widetilde{\varphi}$, then conditions (10) are valid for $\widetilde{\varphi}_{j}$.

2. The Strang-Fix conditions of order $s, s>0$, for a sequence of functions $\left\{\varphi_{j}\right\}_{j}$ :

$$
\left|\widehat{\varphi_{j}}\left(M^{* j} n+r\right)\right| \leq b_{n}\left|M^{*-j} r\right|^{s}, \quad \forall n \neq \mathbf{0}, \quad \forall r \in D\left(M^{* j}\right) .
$$

This corresponds to the well known Strang-Fix conditions for a non-periodic function $\varphi: D^{\beta} \widehat{\varphi}(n)=0$, for $n \neq \mathbf{0},[\beta]<s, s \in \mathbb{N}$ and the condition that $\widehat{\varphi}$ is boundedly differentiable up to order $s$. Indeed, by Taylor's formula near the point $\xi=n$, we have for $r \in D\left(M^{* j}\right)$

$$
\widehat{\varphi}\left(n+M^{*-j} r\right)=\sum_{[\beta]=s} \frac{s}{\beta !}\left(M^{*-j} r\right)^{\beta} \int_{0}^{1}(1-t)^{s-1} D^{\beta} \widehat{\varphi}\left(n+t M^{*-j} r\right) d t .
$$

Thus, since $\widehat{\varphi_{j}}\left(M^{* j} n+r\right)=\widehat{\varphi}\left(n+M^{*-j} r\right)$, we get $\left|\widehat{\varphi_{j}}\left(M^{* j} n+r\right)\right| \leq C_{s, \varphi}\left|M^{*-j} r\right|^{s}$.

For error estimates in the non-periodic case (see, e.g., [22]), the following additional assumption helps:

$$
\sum_{n \in \mathbb{Z}^{d}}\left|D^{\beta} \widehat{\varphi}(n+\xi)\right|^{q} \leq B_{s}, \quad \forall \xi \in \mathbb{R}^{d},[\beta]<s .
$$

A periodic analog of this condition is $\left\{b_{n}\right\}_{n} \in \ell_{q}$.

3. The weak compatibility conditions of order $s, s>0$, for $\left\{\varphi_{j}\right\}_{j}$ and $\left\{\widetilde{\varphi}_{j}\right\}_{j}$ :

$$
\left|1-\widehat{\varphi_{j}}(r) \overline{\widehat{\varphi_{j}}(r)}\right| \leq b_{0}\left|M^{*-j} r\right|^{s}, \quad \forall r \in D\left(M^{* j}\right), \quad \forall j \in \mathbb{N} .
$$

This corresponds to the following non-periodic condition: $D^{\beta}(1-\widehat{\varphi} \overline{\widehat{\varphi}})(\mathbf{0})=0,[\beta]<s, s \in \mathbb{N}$, and condition that $\hat{\varphi} \overline{\widehat{\varphi}}$ is boundedly differentiable up to order $s$. Indeed, by Taylor's formula near the point $\xi=0$,

$$
\widehat{\varphi}\left(M^{*-j} r\right) \overline{\widetilde{\widetilde{\varphi}}\left(M^{*-j} r\right)}=1+\sum_{[\beta]=s} \frac{s}{\beta !}\left(M^{*-j} r\right)^{\beta} \int_{0}^{1}(1-t)^{s-1} D^{\beta} \widehat{\widehat{\varphi}} \overline{\widetilde{\varphi}}\left(t M^{*-j} r\right) d t .
$$

Thus, since $\widehat{\varphi}\left(M^{*-j} r\right) \overline{\overline{\widetilde{\varphi}}\left(M^{*-j} r\right)}=\widehat{\varphi_{j}}(r) \overline{\widehat{\widetilde{\varphi}_{j}}(r)}$, we have $\left|1-\widehat{\varphi_{j}}(r) \overline{\widetilde{\varphi}_{j}(r)}\right| \leq C_{s, \widetilde{\varphi}}\left|M^{*-j} r\right|^{s}$.

4. The conditions on the uniform boundedness of the Fourier coefficients of $\left\{\varphi_{j}\right\}_{j}$ :

$$
\max _{k \in D\left(M^{* j}\right)}\left|\widehat{\varphi_{j}}(k)\right| \leq C_{\varphi}, \quad \forall j \in \mathbb{N} .
$$

\section{Error estimates for $Q_{j}\left(f, \varphi_{j}, \widetilde{\varphi}_{j}\right)$}

\subsection{Estimates in terms of the Fourier coefficients}

First we establish two utility lemmas. For convenience, in the proofs of these lemmas we will use the following notation

$$
C_{d}:=\max _{x \in\left[-\frac{1}{2}, \frac{1}{2}\right]^{d}} \theta_{1}(x)=\left(1+\frac{d}{4}\right)^{\frac{1}{2}} .
$$


Lemma 1 Let $1 \leq q \leq \infty, 1 / p+1 / q=1, j \in \mathbb{N}, \alpha \geq 0,\left\{\widetilde{\varphi}_{j}\right\}_{j}$ be a sequence of periodic distributions whose Fourier coefficients satisfy condition (10) with parameter $N \geq 0$. Suppose $f \in A_{q}^{\gamma}$, where $\gamma>N+d / p$ for $q \neq 1$ or $\gamma \geq N$ for $q=1$. Then, if $1 \leq q<\infty$

$$
\left(\sum_{r \in D\left(M^{* j}\right)}\left|\sum_{n \neq 0} \widehat{f}\left(r+M^{* j} n\right) \overline{\widetilde{\varphi}_{j}\left(r+M^{* j} n\right)}\right|^{q} \theta_{\alpha}^{q}(r)\right)^{\frac{1}{q}} \leq C_{L e m}\left\|M^{* j}\right\|^{\alpha}\left\|M^{*-j}\right\|^{\gamma}\|f\|_{A_{q}^{\gamma}, j}^{O u t},
$$

and if $q=\infty$

$$
\sup _{r \in D\left(M^{* j}\right)}\left|\sum_{n \neq 0} \widehat{f}\left(r+M^{* j} n\right) \overline{\widehat{\widehat{\varphi}_{j}}\left(r+M^{* j} n\right)}\right| \theta_{\alpha}(r) \leq C_{L e m}\left\|M^{* j}\right\|^{\alpha}\left\|M^{*-j}\right\|^{\gamma}\|f\|_{A_{\infty}^{\gamma}, j}^{\text {Out }},
$$

where the constant $C_{\text {Lem }}$ does not depend on $j$ and $f$.

Proof. First, let $1<q<\infty$ and $\alpha=0$. Applying Hölder's inequality, condition (10), and the fact that $\frac{1}{\left|M^{* j} k\right|} \leq \frac{\left\|M^{*-j}\right\|}{|k|}$, we derive

$$
\begin{aligned}
& \sum_{r \in D\left(M^{* j}\right)}\left|\sum_{n \neq \mathbf{0}} \widehat{f}\left(r+M^{* j} n\right) \overline{\widehat{\varphi_{j}}\left(r+M^{* j} n\right)}\right|^{q} \\
& \leq \sum_{r \in D\left(M^{* j}\right)}\left(\sum_{n \neq \mathbf{0}} \frac{1}{\left|r+M^{* j} n\right|^{p(\gamma-N)}}\right)^{\frac{q}{p}} \\
& \times \sum_{n \neq \mathbf{0}}\left|\widehat{f}\left(r+M^{* j} n\right)\right|^{q}\left|\widehat{\widehat{\varphi}_{j}}\left(r+M^{* j} n\right)\right|^{q}\left|r+M^{* j} n\right|^{q(\gamma-N)} \\
& \leq \max _{r \in D\left(M^{* j}\right)}\left(\sum_{n \neq \mathbf{0}} \frac{1}{\left|r+M^{* j} n\right|^{p(\gamma-N)}}\right)^{\frac{q}{p}} \sum_{k \notin D\left(M^{* j}\right)}|\widehat{f}(k)|^{q}\left|\widehat{\widetilde{\varphi}_{j}}(k)\right|^{q}|k|^{q(\gamma-N)} \\
& \leq\left\|M^{*-j}\right\|^{(\gamma-N) q} \max _{\xi \in[-1 / 2,1 / 2)^{d}}\left(\sum_{n \neq \mathbf{0}} \frac{1}{|n+\xi|^{p(\gamma-N)}}\right)^{\frac{q}{p}} C_{\widetilde{\varphi}}^{q}\left\|M^{*-j}\right\|^{q N} \sum_{k \notin D\left(M^{* j}\right)}|\widehat{f}(k)|^{q}|k|^{q \gamma} \\
& \leq C_{p, \gamma, N}^{q} C_{\widetilde{\varphi}}^{q}\left\|M^{*-j}\right\|^{q \gamma}\|f\|_{A_{q}^{\gamma}, j}^{q, \text { out }} .
\end{aligned}
$$

Repeating the same steps, the required estimate for $q=\infty$ can be derived. For $q=1$, we have

$$
\begin{aligned}
& \sum_{r \in D\left(M^{* j}\right)}\left|\sum_{n \neq \mathbf{0}} \widehat{f}\left(r+M^{* j} n\right) \overline{\widehat{\widetilde{\varphi}_{j}}\left(r+M^{* j} n\right)}\right| \leq \sum_{k \notin D\left(M^{* j}\right)}\left|\widehat{f}(k) \overline{\widehat{\varphi_{j}}(k)}\right| \\
& \leq C_{\widetilde{\varphi}} \sum_{k \notin D\left(M^{* j}\right)}\left|\widehat { f } ( k ) \left\|\left.M^{*-j} k\right|^{N} \leq 2^{\gamma} C_{\widetilde{\varphi}} \sum_{k \notin D\left(M^{* j}\right)}\left|\widehat{f}(k)\left\|\left.M^{*-j} k\right|^{\gamma} \leq 2^{\gamma} C_{\widetilde{\varphi}}\right\| M^{*-j}\left\|^{\gamma}\right\| f \|_{A_{1}^{\gamma}, j}^{\text {Out }}\right.\right.\right.
\end{aligned}
$$

since $\left|M^{*-j} k\right| \geq \frac{1}{2}$ for $k \notin D\left(M^{* j}\right)$ and $\left|M^{*-j} k\right|^{N} \leq\left(2\left|M^{*-j} k\right|\right)^{N} \leq\left(2\left|M^{*-j} k\right|\right)^{\gamma}$.

To prove the lemma for $\alpha>0$, it is sufficient to note that

$$
\max _{r \in D\left(M^{* j}\right)} \theta_{\alpha}^{q}(r) \leq\left\|M^{* j}\right\|^{q \alpha} \max _{r \in D\left(M^{* j}\right)} \theta_{\alpha}^{q}\left(M^{*-j} r\right) \leq\left\|M^{* j}\right\|^{q \alpha} \max _{x \in\left[-\frac{1}{2}, \frac{1}{2}\right]^{d}} \theta_{\alpha}^{q}(x) \leq C_{d}^{\alpha q}\left\|M^{* j}\right\|^{q \alpha},
$$

and $C_{L e m}=C_{p, \gamma, N} C_{\widetilde{\varphi}} C_{d}^{\alpha}$ for $q>1$ and $C_{L e m}=2^{\gamma} C_{\widetilde{\varphi}} C_{d}^{\alpha}$ for $q=1 . \diamond$ 
Remark 2 The constant $C_{p, \gamma, N}$ in the proof of Lemma 1] can be estimated as follows (see [31, Lemma 1.10])

$$
C_{p, \gamma, N}^{p}=\max _{\xi \in\left[-\frac{1}{2}, \frac{1}{2}\right)^{d}} \sum_{n \neq \mathbf{0}} \frac{1}{|n+\xi|^{p(\gamma-N)}} \leq 2^{p(\gamma-N)} \sum_{v=1}^{d} 2^{v} \frac{1}{v^{p(\gamma-N) / 2}}\left(\begin{array}{l}
d \\
v
\end{array}\right)\left(1+\frac{v}{2(p(\gamma-N)-v)}\right)^{v} .
$$

Lemma 3 Let $1 \leq q \leq \infty, 1 / p+1 / q=1, j \in \mathbb{N}, \alpha \geq 0,\left\{\varphi_{j}\right\}_{j}$ and $\left\{\widetilde{\varphi}_{j}\right\}_{j}$ be sequences of periodic functions and periodic distributions, respectively, whose Fourier coefficients are such that conditions (10), (11), (12), (14) are valid with parameters $N \geq 0, s>0$, and $b=\left\{b_{n}\right\}_{n} \in \ell_{q}^{\alpha}$. Suppose $f \in A_{q}^{\gamma}$, where $\gamma \geq \alpha$ and $\gamma>N+d / p$ for $q \neq 1$ or $\gamma \geq N$ for $q=1$,

$$
I:= \begin{cases}\left(\sum_{n \in \mathbb{Z}^{d}}\left|\widehat{f}(n)-\sum_{l \in \mathbb{Z}^{d}} \widehat{f}\left(n+M^{* j} l\right) \overline{\widehat{\widetilde{\varphi}}_{j}\left(n+M^{* j} l\right)} \widehat{\varphi_{j}}(n)\right|^{q} \theta_{\alpha}^{q}(n)\right)^{\frac{1}{q}}, & \text { if } q<\infty, \\ \sup _{n \in \mathbb{Z}^{d}}\left|\widehat{f}(n)-\sum_{l \in \mathbb{Z}^{d}} \widehat{f}\left(n+M^{* j} l\right) \widehat{\widehat{\widetilde{\varphi}_{j}}\left(n+M^{* j} l\right) \widehat{\varphi_{j}}}(n)\right| \theta_{\alpha}(n), & \text { if } q=\infty .\end{cases}
$$

Then

$$
I \leq C_{1}\left\|M^{* j}\right\|^{\alpha}\left\|M^{*-j}\right\|^{s}\|f\|_{A_{q}^{s+\alpha}, j}^{I n}+C_{2}\left\|M^{* j}\right\|^{\alpha}\left\|M^{*-j}\right\|^{\gamma}\|f\|_{A_{q}^{\gamma}, j}^{O u t},
$$

where the constants $C_{1}$ and $C_{2}$ do not depend on $j$ and $f$.

Proof. First, we consider the case $q<\infty$. Set

$$
J:=I^{q}=\sum_{n \in \mathbb{Z}^{d}}\left|\widehat{f}(n)-\sum_{l \in \mathbb{Z}^{d}} \widehat{f}\left(n+M^{* j} l\right) \overline{\widehat{\widetilde{\varphi}}_{j}\left(n+M^{* j} l\right)} \widehat{\varphi_{j}}(n)\right|^{q} \theta_{\alpha}^{q}(n)
$$

and split the sum $J$ into two parts such that

$$
J=\sum_{n \in D\left(M^{* j}\right)}+\sum_{n \notin D\left(M^{* j}\right)}:=J_{0}+J_{1} .
$$

Estimating $J_{0}$, we derive

$$
\begin{aligned}
& J_{0}=\sum_{n \in D\left(M^{* j}\right)}\left|\widehat{f}(n)-\widehat{f}(n) \overline{\widehat{\widehat{\varphi}_{j}}(n)} \widehat{\widehat{\varphi}_{j}}(n)-\sum_{l \neq \mathbf{0}} \widehat{f}\left(n+M^{* j} l\right) \overline{\widehat{\widetilde{\varphi}}_{j}\left(n+M^{* j} l\right)} \widehat{\varphi_{j}}(n)\right|^{q} \theta_{\alpha}^{q}(n) \\
& \leq 2^{\frac{q}{p}} \sum_{n \in D\left(M^{* j}\right)}\left|\widehat{f}(n)-\widehat{f}(n) \overline{\widehat{\widetilde{\varphi}_{j}}(n)} \widehat{\varphi_{j}}(n)\right|^{q} \theta_{\alpha}^{q}(n) \\
& \quad+2^{\frac{q}{p}} \sum_{n \in D\left(M^{* j}\right)}\left|\sum_{l \neq \mathbf{0}} \widehat{f}\left(n+M^{* j} l\right) \overline{\widehat{\widetilde{\varphi}}_{j}\left(n+M^{* j} l\right)} \widehat{\varphi_{j}}(n)\right|^{q} \theta_{\alpha}^{q}(n)=: J_{00}+J_{01} .
\end{aligned}
$$

For the first term $J_{00}$, by the compatibility conditions for $\varphi_{j}$ and $\widetilde{\varphi}_{j}$ of order $s$, see (12), we get

$$
\begin{aligned}
J_{00} & =2^{\frac{q}{p}} \sum_{n \in D\left(M^{* j}\right)}|\widehat{f}(n)|^{q}\left|1-\overline{\widehat{\varphi_{j}}(n)} \widehat{\varphi_{j}}(n)\right|^{q} \theta_{\alpha}^{q}(n) \\
& \leq 2^{\frac{q}{p}} b_{0}^{q}\left\|M^{*-j}\right\|^{q s} \sum_{n \in D\left(M^{* j}\right)}|\widehat{f}(n)|^{q}|n|^{q s} \theta_{\alpha}^{q}(n) \leq 2^{\frac{q}{p}} b_{0}^{q}\left\|M^{*-j}\right\|^{q s}\|f\|_{A_{q}^{s+\alpha}, j}^{q, I n} .
\end{aligned}
$$


For the second term $J_{01}$, by Lemma 1 and (14), we have

$$
\begin{aligned}
J_{01} & =2^{\frac{q}{p}} \sum_{n \in D\left(M^{* j}\right)}\left|\sum_{l \neq \mathbf{0}} \widehat{f}\left(n+M^{* j} l\right) \widehat{\widehat{\widehat{\varphi}_{j}}\left(n+M^{* j} l\right)} \widehat{\varphi_{j}}(n)\right|^{q} \theta_{\alpha}^{q}(n) \\
& \leq 2^{\frac{q}{p}} \max _{n \in D\left(M^{* j}\right)}\left|\widehat{\varphi_{j}}(n)\right|^{q} \sum_{n \in D\left(M^{* j}\right)}\left|\sum_{l \neq \mathbf{0}} \widehat{f}\left(n+M^{* j} l\right) \widehat{\widehat{\widetilde{\varphi}}_{j}\left(n+M^{* j} l\right)}\right|^{q} \theta_{\alpha}^{q}(n) \\
& \leq 2^{\frac{q}{p}} C_{\varphi}^{q} C_{L e m}^{q}\left\|M^{* j}\right\|^{q \alpha}\left\|M^{*-j}\right\|^{q \gamma}\|f\|_{A_{q}^{\gamma}, j}^{q, \text { Out }} .
\end{aligned}
$$

Thus, the term $J_{0}$ is estimated by

$$
J_{0} \leq 2^{\frac{q}{p}}\left(b_{0}^{q}\left\|M^{*-j}\right\|^{q s}\|f\|_{A_{q}^{s+\alpha}, j}^{q, I n}+C_{\varphi}^{q} C_{L e m}^{q}\left\|M^{* j}\right\|^{q \alpha}\left\|M^{*-j}\right\|^{q \gamma}\|f\|_{A_{q}^{\gamma}, j}^{q, \text { Out }}\right) .
$$

Consider $J_{1}$. By Minkowski's inequality, we get

$$
\begin{aligned}
& J_{1}:= \sum_{n \notin D\left(M^{* j}\right)}\left|\widehat{f}(n)-\widehat{f}(n) \overline{\widehat{\widehat{\varphi}_{j}}(n)} \widehat{\widehat{\varphi_{j}}}(n)-\sum_{l \neq \mathbf{0}} \widehat{f}\left(n+M^{* j} l\right) \overline{\widehat{\widehat{\varphi}_{j}}\left(n+M^{* j} l\right)} \widehat{\varphi_{j}}(n)\right|^{q} \theta_{\alpha}^{q}(n) \\
& \leq 2^{\frac{q}{p}} \sum_{n \notin D\left(M^{* j}\right)}\left|\widehat{f}(n)-\widehat{f}(n) \overline{\widehat{\widetilde{\varphi}_{j}}(n)} \widehat{\varphi_{j}}(n)\right|^{q} \theta_{\alpha}^{q}(n) \\
& \quad+2^{\frac{q}{p}} \sum_{n \notin D\left(M^{* j}\right)} \mid \sum_{l \neq \mathbf{0}} \widehat{f}\left(n+M^{* j} l\right) \widehat{\left.\widehat{\widetilde{\varphi}_{j}}\left(n+M^{* j} l\right) \widehat{\varphi_{j}}(n)\right|^{q} \theta_{\alpha}^{q}(n)=: J_{10}+J_{11} .}
\end{aligned}
$$

Again, by Minkowski's inequality,

$$
J_{10} \leq 2^{\frac{2 q}{p}} \sum_{n \notin D\left(M^{* j}\right)}|\widehat{f}(n)|^{q} \theta_{\alpha}^{q}(n)+2^{\frac{2 q}{p}} \sum_{n \notin D\left(M^{* j}\right)}\left|\widehat{f}(n) \overline{\widehat{\bar{\varphi}_{j}}(n)} \widehat{\varphi_{j}}(n)\right|^{q} \theta_{\alpha}^{q}(n)=: J_{100}+J_{101} .
$$

For the sum $J_{100}$, using the inequality $\frac{1}{|n|} \leq \frac{\left\|M^{*-j}\right\|}{\left|M^{*-j} n\right|}$, we derive

$$
\begin{aligned}
J_{100} & =2^{\frac{2 q}{p}} \sum_{n \notin D\left(M^{* j}\right)} \frac{|n|^{q(\gamma-\alpha)}}{|n|^{q(\gamma-\alpha)}}|\widehat{f}(n)|^{q} \theta_{\alpha}^{q}(n) \leq 2^{\frac{2 q}{p}}\|f\|_{A_{q}^{\gamma}, j}^{q, \text { Out }} \max _{n \notin D\left(M^{* j}\right)} \frac{1}{|n|^{q(\gamma-\alpha)}} \\
& \leq 2^{\frac{2 q}{p}}\|f\|_{A_{q}^{\gamma}, j}^{q, \text { Out }}\left\|M^{*-j}\right\|^{q(\gamma-\alpha)} \max _{n \notin D\left(M^{* j}\right)} \frac{1}{\left|M^{*-j} n\right|^{q(\gamma-\alpha)}} \\
& \leq 2^{\frac{2 q}{p}} 2^{q(\gamma-\alpha)}\left\|M^{*-j}\right\|^{q(\gamma-\alpha)}\|f\|_{A_{q}^{\gamma}, j}^{q, \text { out }}
\end{aligned}
$$

where the last inequality is valid since for $n \notin D\left(M^{* j}\right)$ we have $\left|M^{*-j} n\right|>1 / 2$.

Consider $J_{101}$. By the Strang-Fix conditions (11) and inequalities (5), (6) and (15), for any $n=M^{* j} k+r$ with $k \neq \mathbf{0}$ and $r \in D\left(M^{* j}\right)$, we obtain

$$
\left|\widehat{\varphi_{j}}(n)\right|^{q} \theta_{\alpha}^{q}(n) \leq b_{k}^{q}\left|M^{*-j} r\right|^{q s}\left\|M^{* j}\right\|^{\alpha q} \theta_{\alpha}^{q}(k) \theta_{\alpha}^{q}\left(M^{*-j} r\right) \leq\|b\|_{\ell_{\infty}^{\alpha}}^{q} C_{d}^{q(s+\alpha)}\left\|M^{* j}\right\|^{\alpha q} .
$$


Therefore,

$$
\begin{aligned}
J_{101} & =2^{\frac{2 q}{p}} \sum_{n \notin D\left(M^{* j}\right)}\left|\widehat{f}(n) \overline{\widehat{\widetilde{\varphi}}_{j}(n)} \widehat{\varphi_{j}}(n)\right|^{q} \theta_{\alpha}^{q}(n) \\
& =2^{\frac{2 q}{p}}\|b\|_{\ell_{\infty}^{\alpha}}^{q} C_{d}^{q(s+\alpha)}\left\|M^{* j}\right\|^{\alpha q} \sum_{n \notin D\left(M^{* j}\right)}\left|\widehat{f}(n) \overline{\widehat{\widetilde{\varphi}}_{j}(n)}\right|^{q} \\
& \leq 2^{\frac{2 q}{p}}\|b\|_{\ell_{\infty}^{\alpha}}^{q} C_{d}^{q(s+\alpha)}\left\|M^{* j}\right\|^{\alpha q} C_{\widetilde{\varphi}}^{q}\left\|M^{*-j}\right\|^{N q} \sum_{n \notin D\left(M^{* j}\right)}|\widehat{f}(n)|^{q}|n|^{q N} \\
& =2^{\frac{2 q}{p}}\|b\|_{\ell_{\infty}^{\alpha}}^{q} C_{d}^{q(s+\alpha)} C_{\widetilde{\varphi}}^{q}\left\|M^{* j}\right\|^{\alpha q}\left\|M^{*-j}\right\|^{N q} \sum_{n \notin D\left(M^{* j}\right)}|\widehat{f}(n)|^{q} \frac{|n|^{q \gamma}}{|n|^{q(\gamma-N)}} \\
& \leq 2^{\frac{2 q}{p}}\|b\|_{\ell_{\infty}^{\alpha}}^{q} C_{d}^{q(s+\alpha)} C_{\widetilde{\varphi}}^{q}\left\|M^{* j}\right\|^{\alpha q}\left\|M^{*-j}\right\|^{N q} \sum_{n \notin D\left(M^{* j}\right)}|\widehat{f}(n)|^{q}|n|^{q \gamma} \frac{\left\|M^{*-j}\right\|^{q(\gamma-N)}}{\left|M^{*-j} n\right|^{q(\gamma-N)}} \\
& \leq 2^{\frac{2 q}{p}} 2^{q(\gamma-N)}\|b\|_{\ell_{\infty}^{\alpha}}^{q} C_{d}^{q(s+\alpha)} C_{\widetilde{\varphi}}^{q}\left\|M^{* j}\right\|^{\alpha q}\left\|M^{*-j}\right\|^{q \gamma}\|f\|_{A_{q}^{\gamma}, j}^{q u t},
\end{aligned}
$$

where the last inequality is valid because $\left|M^{*-j} n\right|>1 / 2$ whenever $n \notin D\left(M^{* j}\right)$, and, therefore,

$$
\frac{1}{\left|M^{*-j} n\right|^{q(\gamma-N)}} \leq \max _{n \notin D\left(M^{* j}\right)} \frac{1}{\left|M^{*-j} n\right|^{q(\gamma-N)}}=2^{q(\gamma-N)} .
$$

Combining the estimates for $J_{100}$ and $J_{101}$, we get

$$
J_{10} \leq 2^{\frac{2 q}{p}}\left(2^{q(\gamma-\alpha)}+2^{q(\gamma-N)}\|b\|_{\ell_{\infty}^{\alpha}}^{q} C_{d}^{q(s+\alpha)} C_{\widetilde{\varphi}}^{q}\right)\left\|M^{* j}\right\|^{q \alpha}\left\|M^{*-j}\right\|^{q \gamma}\|f\|_{A_{q}^{\gamma}, j}^{q, \text { ut }} .
$$

Now, let us estimate $J_{11}$. By Minkowski's inequality,

$$
\begin{aligned}
& J_{11}=2^{\frac{q}{p}} \sum_{n \notin D\left(M^{* j}\right)}\left|\sum_{l \neq \mathbf{0}} \widehat{f}\left(n+M^{* j} l\right) \widehat{\widehat{\widehat{\varphi}_{j}}\left(n+M^{* j} l\right)} \widehat{\varphi_{j}}(n)\right|^{q} \theta_{\alpha}^{q}(n) \\
& =2^{\frac{q}{p}} \sum_{r \in D\left(M^{* j}\right)} \sum_{k \neq \mathbf{0}}\left|\widehat{\varphi_{j}}\left(r+M^{* j} k\right)\right|^{q}\left|\sum_{l \neq \mathbf{0}} \widehat{f}\left(r+M^{* j}(k+l)\right) \overline{\widehat{\widetilde{\varphi}}_{j}\left(r+M^{* j}(k+l)\right)}\right|^{q} \theta_{\alpha}^{q}\left(r+M^{* j} k\right) \\
& =2^{\frac{2 q}{p}} \sum_{r \in D\left(M^{* j}\right)} \sum_{k \neq \mathbf{0}}\left|\widehat{\varphi_{j}}\left(r+M^{* j} k\right)\right|^{q}\left|\widehat{f}(r) \overline{\widehat{\varphi}_{j}(r)}\right|^{q} \theta_{\alpha}^{q}\left(r+M^{* j} k\right) \\
& +2^{\frac{2 q}{p}} \sum_{r \in D\left(M^{* j}\right)} \sum_{k \neq \mathbf{0}}\left|\widehat{\varphi_{j}}\left(r+M^{* j} k\right)\right|^{q}\left|\sum_{\substack{l \neq \mathbf{0} \\
l \neq-k}} \widehat{f}\left(r+M^{* j}(k+l)\right) \overline{\widehat{\widehat{\varphi}_{j}}\left(r+M^{* j}(k+l)\right)}\right|^{q} \theta_{\alpha}^{q}\left(r+M^{* j} k\right) \\
& =: J_{110}+J_{111}
\end{aligned}
$$

Using the Strang-Fix conditions (11), inequalities (5), (6) and conditions (10), we obtain

$$
\begin{aligned}
J_{110} & \leq 2^{\frac{2 q}{p}} \sum_{r \in D\left(M^{* j}\right)}\left|M^{*-j} r\right|^{s q}\left|\widehat{f}(r) \overline{\widehat{\widetilde{\varphi}}_{j}(r)}\right|^{q} \theta_{\alpha}^{q}(r) \sum_{k \neq \mathbf{0}} b_{k}^{q} \theta_{\alpha}^{q}\left(M^{* j} k\right) \\
& \leq 2^{\frac{2 q}{p}} C_{\widetilde{\varphi}}^{q}\left\|M^{*-j}\right\|^{s q} \sum_{r \in D\left(M^{* j}\right)}|r|^{s q}|\widehat{f}(r)|^{q} \theta_{\alpha}^{q}(r)\left\|M^{* j}\right\|^{\alpha q}\|b\|_{\ell_{q}^{\alpha}}^{q} \\
& \leq 2^{\frac{2 q}{p}} C_{\widetilde{\varphi}}^{q}\|b\|_{\ell_{q}^{\alpha}}^{q}\left\|M^{* j}\right\|^{\alpha q}\left\|M^{*-j}\right\|^{s q}\|f\|_{A_{q}^{s+\alpha}, j}^{q, I n} .
\end{aligned}
$$


For the second term $J_{111}$, using Lemma 1 with $\alpha=0$, the Strang-Fix conditions (11) and (10), inequalities (5) and (6), we get

$$
\begin{aligned}
& J_{111}:=2^{\frac{2 q}{p}} \sum_{r \in D\left(M^{* j}\right)} \sum_{k \neq \mathbf{0}}\left|\widehat{\varphi_{j}}\left(r+M^{* j} k\right)\right|^{q}\left|\sum_{\substack{l \neq \mathbf{0} \\
l \neq-k}} \widehat{f}\left(r+M^{* j}(k+l)\right) \overline{\widehat{\widetilde{\varphi}}_{j}\left(r+M^{* j}(k+l)\right)}\right|^{q} \theta_{\alpha}^{q}\left(r+M^{* j} k\right) \\
& \leq 2^{\frac{2 q}{p}} \sum_{r \in D\left(M^{* j}\right)} \sum_{k \neq \mathbf{0}} b_{k}^{q}\left|M^{*-j} r\right|^{s q}\left\|M^{* j}\right\|^{\alpha q} \theta_{\alpha}^{q}\left(M^{*-j} r\right) \theta_{\alpha}^{q}(k) \\
& \times\left|\sum_{\substack{l \neq \mathbf{0} \\
l \neq-k}} \widehat{f}\left(r+M^{* j}(k+l)\right) \overline{\widehat{\widehat{\varphi}}_{j}\left(r+M^{* j}(k+l)\right)}\right|^{q} \\
& \leq 2^{\frac{2 q}{p}} C_{d}^{q(s+\alpha)}\left\|M^{* j}\right\|^{\alpha q} \sum_{k \neq \mathbf{0}} b_{k}^{q} \theta_{\alpha}^{q}(k) \sum_{r \in D\left(M^{* j}\right)}\left|\sum_{\substack{l \neq \mathbf{0} \\
l \neq-k}} \widehat{f}\left(r+M^{* j}(k+l)\right) \overline{\widehat{\varphi}_{j}\left(r+M^{* j}(k+l)\right)}\right|^{q} \\
& \leq 2^{\frac{2 q}{p}} C_{d}^{q(s+\alpha)}\|b\|_{\ell_{q}^{\alpha}}^{q} C_{L e m}^{q}\left\|M^{* j}\right\|^{\alpha q}\left\|M^{*-j}\right\|^{q \gamma}\|f\|_{A_{q}^{\gamma}, j}^{q, O u t} .
\end{aligned}
$$

Thus, combining the estimates for $J_{11}, J_{110}$, and $J_{111}$, we derive

$$
\begin{aligned}
J_{11} \leq & 2^{\frac{2 q}{p}} C_{\widetilde{\varphi}}^{q}\|b\|_{\ell_{q}^{\alpha}}^{q}\left\|M^{* j}\right\|^{q \alpha}\left\|M^{*-j}\right\|^{s q}\|f\|_{A_{q}^{s+\alpha}, j}^{q, I n} \\
& +2^{\frac{2 q}{p}} C_{d}^{q(s+\alpha)}\|b\|_{\ell_{q}^{\alpha}}^{q} C_{L e m}^{q}\left\|M^{* j}\right\|^{q \alpha}\left\|M^{*-j}\right\|^{q \gamma}\|f\|_{A_{q}^{\gamma}, j}^{q, \text { Out }} .
\end{aligned}
$$

Finally, the estimates for $J_{1}, J_{10}$, and $J_{11}$ yield

$$
\begin{aligned}
J_{1} \leq C_{J_{10}} & \left\|M^{* j}\right\|^{q \alpha}\left\|M^{*-j}\right\|^{q \gamma}\|f\|_{A_{q}^{\gamma}, j}^{q, \text { out }} \\
& +C_{J_{110}}\left\|M^{* j}\right\|^{q \alpha}\left\|M^{*-j}\right\|^{s q}\|f\|_{A_{q}^{s+\alpha}, j}^{q, \text { In }}+C_{J_{111}}\left\|M^{* j}\right\|^{q \alpha}\left\|M^{*-j}\right\|^{q \gamma}\|f\|_{A_{q}^{\gamma}, j}^{q, \text { out }},
\end{aligned}
$$

which together with (17) implies

$$
\begin{aligned}
& \sum_{n \in \mathbb{Z}^{d}}\left|\widehat{f}(n)-\sum_{l \in \mathbb{Z}^{d}} \widehat{f}\left(n+M^{* j} l\right) \widehat{\widehat{\widehat{\varphi}_{j}}\left(n+M^{* j} l\right)} \widehat{\varphi_{j}}(n)\right|^{q} \theta_{\alpha}^{q}(n) \\
& \leq 2^{\frac{q}{p}} b_{0}^{q}\left\|M^{*-j}\right\|^{q s}\|f\|_{A_{q}^{s+\alpha}, j}^{q, I n}+2^{\frac{q}{p}} C_{\varphi}^{q} C_{L e m}^{q}\left\|M^{* j}\right\|^{q \alpha}\left\|M^{*-j}\right\|^{q \gamma}\|f\|_{A_{q}^{\gamma}, j}^{q, \text { out }} \\
& \quad+C_{J_{110}}\left\|M^{* j}\right\|^{q \alpha}\left\|M^{*-j}\right\|^{s q}\|f\|_{A_{q}^{s+\alpha}, j}^{q, I n}+\left(C_{J_{10}}+C_{J_{111}}\right)\left\|M^{* j}\right\|^{q \alpha}\left\|M^{*-j}\right\|^{q \gamma}\|f\|_{A_{q}^{\gamma}, j}^{q, \text { Out }} \\
& \leq C_{1}\left\|M^{* j}\right\|^{q \alpha}\left\|M^{*-j}\right\|^{q s}\|f\|_{A_{q}^{s+\alpha}, j}^{q, I n}+C_{2}\left\|M^{* j}\right\|^{q \alpha}\left\|M^{*-j}\right\|^{q \gamma}\|f\|_{A_{q}^{\gamma}, j}^{q, \text { Out }},
\end{aligned}
$$

which completes the proof of (16) for the case $q<\infty$.

If now $q=\infty$, then repeating step by step all above estimates, one can easily obtain (16) for this case. $\diamond$

Remark 4 Analyzing the proof of Lemma 3, it is not difficult see that

$$
C_{1}=2^{\frac{q}{p}} b_{0}^{q}+2^{\frac{2 q}{p}} C_{\widetilde{\varphi}}^{q}\|b\|_{\ell_{q}^{\alpha}}^{q}, \quad C_{2} \leq C_{2}^{\prime}\|b\|_{\ell_{\infty}}+C_{2}^{\prime \prime}\|b\|_{\ell_{q}^{\alpha}}^{q},
$$

where $C_{2}^{\prime}$ and $C_{2}^{\prime \prime}$ do not depend on $b$. 
Remark 5 If under the assumptions of Lemma 3 the functions $\varphi_{j}$ are trigonometric polynomials such that spec $\varphi_{j} \subset M^{* j} B_{R}(\mathbf{0})$, then the assumption $\left\{b_{n}\right\}_{n} \in \ell_{q}^{\alpha}$ is not required. Indeed, there exists a finite set $\Omega \subset \mathbb{Z}^{d}$, depending only on $R$ and $d$, such that $\widehat{\varphi_{j}}\left(M^{* j} n+r\right) \neq 0$ for at least one $r \in D\left(M^{* j}\right)$ only for $n \in \Omega$. Analyzing the proof of Lemma 3, we see that $\|b\|_{\ell_{q}^{\alpha}}$ can be replaced by $C_{R, \alpha}\|b\|_{\ell_{\infty}}$.

Theorem 6 Let $1 \leq q \leq \infty, 1 / p+1 / q=1, j \in \mathbb{N}, \alpha \geq 0$, and let $\left\{\varphi_{j}\right\}_{j},\left\{\widetilde{\varphi}_{j}\right\}_{j}$ be a sequence of periodic functions in $L_{p}$ and periodic distributions, respectively, whose Fourier coefficients are such that conditions (10), (11), (12), (14) are valid with parameters $N \geq 0, s>0$, and $\left\{b_{n}\right\}_{n} \in \ell_{q}^{\alpha}$. Suppose $f \in A_{q}^{\gamma}$, where $\gamma>\alpha$ and $\gamma>N+d / p$ for $q \neq 1$ or $\gamma \geq N$ for $q=1$. Then

$$
\left\|f-Q_{j}\left(f, \varphi_{j}, \widetilde{\varphi}_{j}\right)\right\|_{A_{q}^{\alpha}} \leq C\left\|M^{* j}\right\|^{2 \alpha}\left\|M^{*-j}\right\|^{\min \{\gamma, s+\alpha\}}\|f\|_{A_{q}^{\gamma}}
$$

where $C$ does not depend on $j$ and $f$.

In addition, if $M$ is an isotropic matrix and $\lambda$ is its eigenvalue, then

$$
\left\|f-Q_{j}\left(f, \varphi_{j}, \widetilde{\varphi}_{j}\right)\right\|_{A_{q}^{\alpha}} \leq C^{\prime}|\lambda|^{-j \min \{\gamma-2 \alpha, s-\alpha\}}\|f\|_{A_{q}^{\gamma}}
$$

where $C^{\prime}$ does not depend on $j$ and $f$.

Proof. First of all we mention that the inner product $\left\langle f, \widetilde{\varphi}_{j}\left(\cdot-M^{-j} k\right)\right\rangle$ has meaning under our assumptions, and hence the operator $Q_{j}\left(f, \varphi_{j}, \widetilde{\varphi}_{j}\right)$ is well defined. Taking into account that

$$
\sum_{k \in D\left(M^{j}\right)} e^{2 \pi i\left(k, M^{*-j} r\right)}=\left\{\begin{array}{lll}
m^{j}, & \text { if } & r \equiv \mathbf{0}\left(\bmod M^{* j}\right), \\
0, & \text { if } & r \neq \mathbf{0}\left(\bmod M^{* j}\right),
\end{array}\right.
$$

we derive the following representation for the Fourier coefficients of $g:=f-Q_{j}\left(f, \varphi_{j}, \widetilde{\varphi}_{j}\right)$ :

$$
\begin{aligned}
\widehat{g}(n) & =\widehat{f}(n)-\frac{1}{m^{j}} \sum_{k \in D\left(M^{j}\right)}\left\langle f, \widetilde{\varphi}_{j}\left(\cdot-M^{-j} k\right)\right\rangle \widehat{\varphi_{j}}(n) e^{-2 \pi i\left(n, M^{-j} k\right)} \\
& =\widehat{f}(n)-\frac{1}{m^{j}} \widehat{\varphi_{j}}(n) \sum_{l \in \mathbb{Z}^{d}} \widehat{f}(l) \overline{\widehat{\varphi}_{j}}(l) \sum_{k \in D^{\prime}\left(M^{j}\right)} e^{2 \pi i\left(k, M^{*-j}(l-n)\right)} \\
& =\widehat{f}(n)-\widehat{\varphi_{j}}(n) \sum_{l \in \mathbb{Z}^{d}} \widehat{f}\left(n+M^{* j} l\right) \widehat{\widehat{\widetilde{\varphi}_{j}}\left(n+M^{* j} l\right) .}
\end{aligned}
$$

Using this together with Lemma 3 taking into account that $\left\|M^{* j}\right\|^{-1} \leq\left\|M^{*-j}\right\|$, we obtain

$$
\left\|f-Q_{j}\left(f, \varphi_{j}, \widetilde{\varphi}_{j}\right)\right\|_{A_{q}^{\alpha}} \leq C_{1}\left\|M^{* j}\right\|^{2 \alpha}\left\|M^{*-j}\right\|^{s+\alpha}\|f\|_{A_{q}^{s+\alpha}}+C_{2}\left\|M^{* j}\right\|^{\alpha}\left\|M^{*-j}\right\|^{\gamma}\|f\|_{A_{q}^{\gamma}}
$$

Assume first that $s+\alpha \leq \gamma$. Obviously, in this case, $\|f\|_{A_{q}^{s+\alpha}} \leq\|f\|_{A_{q}^{\gamma}}$ and $\left\|M^{*-j}\right\|^{\gamma} \leq\left\|M^{*-j}\right\|^{s+\alpha}$ for all $j$ such that $\left\|M^{*-j}\right\| \leq 1$. By (3), the last inequality is valid for all $j$ which are greater than some appropriate $j_{0}$, and there exists a constant $C_{j_{0}}$ such that $\left\|M^{*-j}\right\|^{\gamma} \leq C_{j_{0}}\left\|M^{*-j}\right\|^{s+\alpha}$ for all $j \leq j_{0}$, which yields (18).

Next, if $s+\alpha>\gamma$, then we set $s^{\prime}=\gamma-\alpha$ and note that all assumptions of Theorem 6 with $s^{\prime}$ instead of $s$ are satisfied. Indeed, we need to check that (11) and (12) are valid for $s^{\prime}$. For low dimensions $(d \leq 4)$, we have $\left|M^{*-j} r\right| \leq 1$ for $r \in D\left(M^{* j}\right)$ and, therefore, (11) and (12) obviously hold. For $d>4$, (11) and (12) are valid for $s^{\prime}$ if we replace the constants $b_{n}$ by $b_{n}\left(\frac{\sqrt{d}}{2}\right)^{s-s^{\prime}}$. Hence, inequality (20) with $s^{\prime}$ instead of $s$ holds and $s^{\prime}+\alpha=\gamma=\min \{s+\alpha, \gamma\}$, which yields (18).

Finally, inequality (19) follows immediately from (18) and relation (4). $\diamond$ 
Note that estimate (19) is actually a generalization of (2). To compare these relations, one has to take into account that (2) was obtained under the following strengthened Strang-Fix conditions of order $s$ for a sequence of fundamental interpolants $\left\{\Lambda_{j}\right\}_{j}$ :

$$
\left|\widehat{\Lambda_{j}}\left(2^{j} n+r\right)\right| \leq b_{n}|r|^{s} 2^{-j(s+\alpha)}, \quad \forall n \neq \mathbf{0}, r \in D\left(M^{* j}\right) .
$$

Analyzing the proof of Lemma 3, it is not difficult to see that under the same strengthened StrangFix conditions for $\left\{\varphi_{j}\right\}_{j}$ instead of (11), we can replace $\min \{\gamma-2 \alpha, s-\alpha\}$ by $\min \{\gamma-\alpha, s\}$ in inequality (19).

Remark 7 If under the assumptions of Theorem [6, the functions $\varphi_{j}$ are trigonometric polynomials such that spec $\varphi_{j} \subset D\left(M^{* j}\right)$, then, analyzing the proof of Lemma 3 , one can easily see that

$$
J_{1}=J_{100}=\sum_{n \notin D\left(M^{* j}\right)}|\widehat{f}(n)|^{q} \theta_{\alpha}^{q}(n) \leq 2^{q(\gamma-\alpha)}\left\|M^{*-j}\right\|^{q(\gamma-\alpha)}\|f\|_{A_{q}^{\gamma}, j}^{q, \text { Out }},
$$

which together with (17) implies

$$
J \leq C_{1}\|f\|_{A_{q}^{s+\alpha}, j}^{q, I n}\left\|M^{*-j}\right\|^{q s}+C_{2} \quad\left\|M^{* j}\right\|^{q \alpha}\left\|M^{*-j}\right\|^{q \gamma}\|f\|_{A_{q}^{\gamma}, j}^{q, O u t} .
$$

It follows that (18) can be replaced by

$$
\left\|f-Q_{j}\left(f, \varphi_{j}, \widetilde{\varphi}_{j}\right)\right\|_{A_{q}^{\alpha}} \leq C\left\|M^{* j}\right\|^{\alpha}\left\|M^{*-j}\right\|^{\min \{\gamma, s+\alpha\}}\|f\|_{A_{q}^{\gamma}}
$$

where $C$ does not depend on $j$ and $f$. In a similar way, relation (19) can be also improved in this case.

Next we need the following embedding properties between the spaces $L_{p}$ and $A_{q}^{0}$.

Proposition 8 If $2 \leq p \leq \infty, 1 / p+1 / q=1$, then $A_{q}^{0} \subset L_{p}$, with

$$
\|f\|_{p} \leq\|f\|_{A_{q}^{0}} \text {. }
$$

Relation (21) is the classical Hausdorff-Young inequality, for its multivariate version see, e.g., [16, p. 174] or [35].

Theorem 9 Let $2 \leq p \leq \infty, 1 / p+1 / q=1,\left\{\varphi_{j}\right\}_{j},\left\{\widetilde{\varphi}_{j}\right\}_{j}, N, s$, and $\gamma$ be as in Theorem [6] with $\alpha=0$ and $f \in A_{q}^{\gamma}$. Then

$$
\left\|f-Q_{j}\left(f, \varphi_{j}, \widetilde{\varphi}_{j}\right)\right\|_{p} \leq C_{1}\left\|M^{*-j}\right\|^{s}\|f\|_{A_{q}^{s}, j}^{I n}+C_{2}\left\|M^{*-j}\right\|^{\gamma}\|f\|_{A_{q}^{\gamma}, j}^{O u t},
$$

where $C_{1}$ and $C_{2}$ do not depend on $j$ and $f$.

If $\vartheta$ is any positive number which is smaller in absolute value than any eigenvalue of $M$, then

$$
\left\|f-Q_{j}\left(f, \varphi_{j}, \widetilde{\varphi}_{j}\right)\right\|_{p} \leq C \vartheta^{-j \min \{s, \gamma\}}\|f\|_{A_{q}^{\gamma}},
$$

where $C$ does not depend on $j$ and $f$. In addition, if $M$ is an isotropic matrix and $\lambda$ is its eigenvalue, then $\vartheta$ can be replaced by $|\lambda|$ in (23).

Proof. Obviously, the operator $Q_{j}\left(f, \varphi_{j}, \widetilde{\varphi}_{j}\right)$ is well defined and belongs to $L_{p}$ under our assumptions. Using (21), one can see that $f \in L_{p}$ and

$$
\left\|f-Q_{j}\left(f, \varphi_{j}, \widetilde{\varphi}_{j}\right)\right\|_{p} \leq\left\|f-Q_{j}\left(f, \varphi_{j}, \widetilde{\varphi}_{j}\right)\right\|_{A_{q}^{0}} .
$$

Analyzing the proof of Theorem 6 and using Lemma 3, we obtain (22). Inequality (23) follows from (24), (18) with $\alpha=0$, and (3). It remains to say that in the case of isoropic matrix $M$, one can use (4) instead of (31). $\diamond$

Note that non-periodic counterparts of the results stated in Theorem 9 were obtained in 22, see Theorems 4, 5 . 
Corollary 10 If under the assumptions of Theorem 9 we have $\widehat{f}(n)=\mathcal{O}\left(|n|^{-\kappa}\right)$, where $\kappa>N+d$, and $M$ is an isotropic matrix, then

$$
\left\|f-Q_{j}\left(f, \varphi_{j}, \widetilde{\varphi}_{j}\right)\right\|_{p}= \begin{cases}\mathcal{O}\left(|\lambda|^{-j s}\right), & s<\kappa-d / q \\ \mathcal{O}\left(j|\lambda|^{-j s}\right), & s=\kappa-d / q \\ \mathcal{O}\left(|\lambda|^{-j\left(\kappa-\frac{d}{q}\right)}\right), & s>\kappa-d / q\end{cases}
$$

where $\lambda$ is an eigenvalue of $M$.

Proof. First, we assume that $s<\kappa-\frac{d}{q}$ and choose $\gamma>N+\frac{d}{p}$ such that $s<\gamma<\kappa-\frac{d}{q}$. Therefore, $s-\kappa<\gamma-\kappa<-\frac{d}{q}$ and both expressions $\|f\|_{A_{q}^{\gamma}, j}^{q, O u t}$ and $\|f\|_{A_{q}^{s}, j}^{q, I n}$, in (22) are finite. Hence, inequality (22) together with (4) yields that

$$
\left\|f-Q_{j}\left(f, \varphi_{j}, \widetilde{\varphi}_{j}\right)\right\|_{p}^{q} \leq C\left\|M^{*-j}\right\|^{q s} \sum_{k \in \mathbb{Z}^{d}}|k|^{(\gamma-\kappa) q}=\mathcal{O}\left(\left\|M^{*-j}\right\|^{q s}\right) .
$$

Now, we assume that $s \geq \kappa-\frac{d}{q}$. Choose $\gamma>N+\frac{d}{p}$ such that $\gamma<\kappa-\frac{d}{q}$. Let us consider the second term in (22). Since $\frac{1}{|n|} \leq \frac{\left\|M^{*-j}\right\|}{\left|M^{*-j} n\right|}$ we get

$$
\begin{aligned}
\left\|M^{*-j}\right\|^{q \gamma}\|f\|_{A_{q}^{\gamma}, j}^{q, \text { Out }} & \leq\left\|M^{*-j}\right\|^{q \gamma} \sum_{n \neq \mathbf{0}} \sum_{r \in D\left(M^{* j}\right)} \frac{1}{\left|M^{* j} n+r\right|^{(\kappa-\gamma) q}} \\
& \leq\left\|M^{*-j}\right\|^{q \gamma} \sum_{n \neq \mathbf{0}} \sum_{r \in D\left(M^{* j}\right)} \max _{\xi \in\left[-\frac{1}{2}, \frac{1}{2}\right)^{d}} \frac{\left\|M^{*-j}\right\|^{q(\kappa-\gamma)}}{|n+\xi|^{(\kappa-\gamma) q}} \\
& \leq C m^{j}\left\|M^{*-j}\right\|^{q \kappa}
\end{aligned}
$$

Next, consider the first term in (22). Then

$$
\left\|M^{*-j}\right\|^{q s}\|f\|_{A_{q}^{s}, j}^{q, I n} \leq C\left\|M^{*-j}\right\|^{q s} \sum_{n \in D\left(M^{* j}\right)}|n|^{(s-\kappa) q} .
$$

It is clear that there exists $A=A(d)>\sqrt{d} / 2$ such that for $|n| \geq A,|x| / 2 \leq|n| \leq 2|x|$ for any $x \in n+\frac{1}{2}[-1,1)^{d}$ (note that anyway $|x|>\widetilde{A}$, for some $\widetilde{A}>0$ ). Therefore, if $s-\kappa \geq 0$, then $|n|^{(s-\kappa) q} \leq 2^{(s-\kappa) q}|x|^{(s-\kappa) q}$, and if $s-\kappa<0$, then $|n|^{(s-\kappa) q} \leq(|x| / 2)^{(s-\kappa) q}$. Thus,

$$
|n|^{(s-\kappa) q} \leq 2^{|s-\kappa| q} \int_{n+\frac{1}{2}[-1,1)^{d}}|x|^{(s-\kappa) q} d x
$$

Hence,

$$
\begin{aligned}
\sum_{n \in D\left(M^{* j}\right)}|n|^{(s-\kappa) q} & =\sum_{|n|<A}|n|^{(s-\kappa) q}+\sum_{|n| \geq A}|n|^{(s-\kappa) q} \leq \widetilde{C}_{1}+2^{|s-\kappa| q} \int_{\widetilde{A}<|x|<\left\|M^{* j}\right\| \sqrt{\frac{d}{4}}}|x|^{(s-\kappa) q} d x \\
& \leq \widetilde{C}_{2} \int_{\widetilde{A}}^{\left\|M^{* j}\right\| \sqrt{\frac{d}{4}}} r^{(s-\kappa) q+d-1} d r \leq\left\{\begin{array}{l}
\widetilde{C}_{3} \log \left\|M^{* j}\right\|, s=\kappa-\frac{d}{q} ; \\
\widetilde{C}_{4}\left\|M^{* j}\right\|^{\left(s-\left(\kappa-\frac{d}{q}\right)\right) q}, s>\kappa-\frac{d}{q} .
\end{array}\right.
\end{aligned}
$$

Overall, for $s=\kappa-\frac{d}{q}$ inequality (22) and above considerations yields that

$$
\left\|f-Q_{j}\left(f, \varphi_{j}, \widetilde{\varphi}_{j}\right)\right\|_{p}^{q} \leq C\left\|M^{*-j}\right\|^{q s}\left(m^{j}\left\|M^{*-j}\right\|^{d}+\log \left\|M^{* j}\right\|\right) .
$$


For $s>\kappa-\frac{d}{q}$ inequality (22) and above considerations yields that

$$
\left\|f-Q_{j}\left(f, \varphi_{j}, \widetilde{\varphi}_{j}\right)\right\|_{p}^{q} \leq C\left(m^{j}\left\|M^{*-j}\right\|^{q \kappa}+\left\|M^{*-j}\right\|^{q s}\left\|M^{* j}\right\|^{\left(s-\left(\kappa-\frac{d}{q}\right)\right) q}\right) .
$$

It remains to note that $m=|\lambda|^{d}$ for isotropic dilation matrix, and using (44), we get the required estimates. $\diamond$

Let $\mathcal{B}=\mathcal{B}_{\delta, R}$ denote the class of sequences of trigonometric polynomials $\varphi_{j}, j \in \mathbb{N}$, such that

$$
\begin{gathered}
\operatorname{spec} \varphi_{j} \subset M^{* j}\left(B_{R}(\mathbf{0}) \backslash \bigcup_{n \in \mathbb{Z}^{d} \backslash\{\mathbf{0}\}} B_{\delta}(n)\right), \\
\left|\widehat{\varphi_{j}}(l)\right| \leq C_{\varphi}^{\prime} \quad \forall j \in \mathbb{N}, \forall l \in \mathbb{Z}^{d},
\end{gathered}
$$

for some positive constants $R, \delta$, and $C_{\varphi}^{\prime}$.

Note that $\left\{\varphi_{j}\right\}_{j}$ belongs to $\mathcal{B}$ whenever (27) is valid and

$$
\operatorname{spec} \varphi_{j} \subset M^{* j}\left([-1+\delta, 1-\delta]^{d}\right), \quad \delta \in(0,1 / 2) .
$$

Definition 11 We say that a sequence of integrable periodic functions $\left\{\varphi_{j}\right\}_{j}$ is strictly compatible with a sequence of periodic distributions $\left\{\widetilde{\varphi}_{j}\right\}_{j}$ if

$$
\widehat{\bar{\varphi}_{j}}(l) \overline{\widehat{\varphi_{j}}(l)}=1, \quad \forall j \in \mathbb{N}, \forall l \in \mathbb{Z}^{d}:\left|M^{*-j} l\right| \leq \delta,
$$

for some $\delta>0$.

Theorem 12 Let $1 \leq q<\infty, 1 / p+1 / q=1,\left\{\widetilde{\varphi}_{j}\right\}_{j}$ be a sequence of periodic distributions satisfying (10) with $N \geq 0$, and a sequence of trigonometric polynomials $\left\{\varphi_{j}\right\}_{j} \in \mathcal{B}$ be strictly compatible with $\left\{\widetilde{\varphi}_{j}\right\}_{j}$ with respect to the parameter $\delta \in(0,1 / 2)$. Suppose $f \in A_{q}^{\gamma}$, where $\gamma>0$ and $\gamma>N+d / p$ for $q \neq 1$ or $\gamma \geq N$ for $q=1$, and $\alpha \in[0, \gamma)$. Then

$$
\left\|f-Q_{j}\left(f, \varphi_{j}, \widetilde{\varphi}_{j}\right)\right\|_{A_{q}^{\alpha}} \leq C\left\|M^{* j}\right\|^{2 \alpha}\left\|M^{*-j}\right\|^{\gamma}\left(\sum_{\left|M^{*-j} r\right| \geq \delta}|r|^{q \gamma}|\widehat{f}(r)|^{q}\right)^{\frac{1}{q}},
$$

and moreover, in the case $p \geq 2$,

$$
\left\|f-Q_{j}\left(f, \varphi_{j}, \widetilde{\varphi}_{j}\right)\right\|_{p} \leq C^{\prime}\left\|M^{*-j}\right\|^{\gamma}\left(\sum_{\left|M^{*-j} r\right| \geq \delta}|r|^{q \gamma}|\widehat{f}(r)|^{q}\right)^{\frac{1}{q}},
$$

where $C, C^{\prime}$ do not depend on $f$ and $j$.

Proof. As in Theorem $\left[6\right.$ the operator $Q_{j}\left(f, \varphi_{j}, \widetilde{\varphi}_{j}\right)$ is well defined under our assumptions. Let us check that for any $s>0$ the Strang-Fix conditions of order $s$ for $\varphi_{j}$ and the weak compatibility conditions for $\varphi_{j}$ and $\widetilde{\varphi}_{j}$ of order $s$ are satisfied. Indeed, let $n \in \mathbb{Z}^{d}, n \neq \mathbf{0}, r \in D\left(M^{* j}\right)$. If $\left|M^{*-j} r\right| \leq \delta$, then $\widehat{\varphi_{j}}\left(M^{* j} n+r\right)=0$; if $\left|M^{*-j} r\right|>\delta$, then, by (27),

$$
\left|\widehat{\varphi_{j}}\left(M^{* j} n+r\right)\right| \leq C_{\varphi}^{\prime} \delta^{-s} \delta^{s} \leq C_{\varphi}^{\prime} \delta^{-s}\left|M^{*-j} r\right|^{s} .
$$

Thus, conditions (11) are satisfied with $b_{n} \equiv C_{\varphi}^{\prime} \delta^{-s}$. Similarly, for every $r \in D\left(M^{* j}\right)$, using (27), (28) and (10), we have

$$
\left|1-\overline{\widehat{\varphi_{j}}(r)} \widehat{\varphi_{j}}(r)\right| \leq\left(1+C_{\varphi}^{\prime} C_{\widetilde{\varphi}}\right) \delta^{-s} \delta^{s} \leq\left(1+C_{\varphi}^{\prime} C_{\widetilde{\varphi}}\right) \delta^{-s}\left|M^{*-j} r\right|^{s}
$$


which implies that (12) is satisfied with $b_{0}=\left(1+C_{\varphi}^{\prime} C_{\widetilde{\varphi}}\right) \delta^{-s}$.

Now, taking into account Remark 5, we see that all assumptions of Lemma 3 are fulfilled with any $s>0$. Analyzing the proof of this lemma, we see that

$$
\begin{aligned}
J & :=\sum_{n \in \mathbb{Z}^{d}}\left|\widehat{f}(n)-\widehat{\varphi_{j}(n)} \sum_{l \in \mathbb{Z}^{d}} \widehat{f}\left(n+M^{* j} l\right) \widehat{\widehat{\widehat{\varphi}_{j}}\left(n+M^{* j} l\right)}\right|^{q} \theta_{\alpha}^{q}(n) \\
& \leq J_{00}+J_{110}+C_{2}\left\|M^{* j}\right\|^{\alpha q}\left\|M^{*-j}\right\|^{q \gamma}\|f\|_{A_{q}^{q}, j}^{q, \text { Out }},
\end{aligned}
$$

Obviously,

$$
\|f\|_{A_{q}^{\gamma}, j}^{q, \text { Out }} \leq 2^{q \gamma} \sum_{\left|M^{*-j} r\right| \geq \delta}|r|^{q \gamma}|\widehat{f}(r)|^{q \gamma} .
$$

Repeating step by step the estimates for $J_{00}$ and $J_{110}$, using (28), (26), and Remark 5 , and taking into account that $\left\|M^{* j}\right\|^{-1} \leq\left\|M^{*-j}\right\|$, we obtain

$$
\begin{aligned}
J_{00} & =2^{q / p} \sum_{r \in D\left(M^{* j}\right)}|\widehat{f}(r)|^{q}\left|1-\overline{\widehat{\widetilde{\varphi}_{j}}(r)} \widehat{\varphi_{j}}(r)\right|^{q} \theta_{\alpha}^{q}(r) \\
& \leq 2^{q(\alpha+1 / p)} b_{0}^{q}\left\|M^{* j}\right\|^{\alpha q}\left\|M^{*-j}\right\|^{(s+\alpha) q} \sum_{\left|M^{*-j} r\right| \geq \delta}|r|^{q(s+\alpha)}|\widehat{f}(r)|^{q}
\end{aligned}
$$

and

$$
\begin{aligned}
J_{110} & =2^{\frac{2 q}{p}} \sum_{r \in D\left(M^{* j}\right)} \sum_{k \neq \mathbf{0}}\left|\widehat{\varphi_{j}}\left(r+M^{* j} k\right)\right|^{q}\left|\widehat{f}(r) \overline{\widehat{\widetilde{\varphi}}_{j}(r)}\right|^{q} \theta_{\alpha}^{q}\left(r+M^{* j} k\right) \\
& \leq 2^{q(\alpha+2 / p)} \delta^{-s} C_{\varphi}^{\prime} C_{\widetilde{\varphi}}^{q} C_{R, \alpha}^{q}\left\|M^{* j}\right\|^{2 \alpha q}\left\|M^{*-j}\right\|^{(s+\alpha) q} \sum_{\left|M^{*-j} r\right| \geq \delta}|r|^{q(s+\alpha)}|\widehat{f}(r)|^{q},
\end{aligned}
$$

where $C_{R, \alpha}$ is the constant from Remark 5. Substituting these relations into (31) and choosing $s=\gamma-\alpha$, we obtain (29).

To prove (30) it remains to set $\alpha=0$ and combine (29) with (21).

Note that relation (30) with $p=\infty$ is a periodic analog of Theorem 15 in 24], which, in turn, is a generalization of Brown's inequality, see [6].

Proposition 13 If under the assumptions of Theorem [12, equality (28) holds for all $l \in D\left(M^{* j}\right)$ and $\operatorname{spec} \varphi_{j} \subset D\left(M^{* j}\right)$, then

$$
\left\|f-Q_{j}\left(f, \varphi_{j}, \widetilde{\varphi}_{j}\right)\right\|_{A_{q}^{\alpha}} \leq C\left\|M^{* j}\right\|^{\alpha}\left\|M^{*-j}\right\|^{\gamma}\left(\sum_{r \notin D\left(M^{* j}\right)}|r|^{q \gamma}|\widehat{f}(r)|^{q}\right)^{\frac{1}{q}},
$$

and moreover, in the case $p \geq 2$,

$$
\left\|f-Q_{j}\left(f, \varphi_{j}, \widetilde{\varphi}_{j}\right)\right\|_{p} \leq C^{\prime}\left\|M^{*-j}\right\|^{\gamma}\left(\sum_{r \notin D\left(M^{* j}\right)}|r|^{q \gamma}|\widehat{f}(r)|^{q}\right)^{\frac{1}{q}},
$$

where $C$ and $C^{\prime}$ do not depend on $f$ and $j$.

Proof. Analyzing the proof of Theorem 12, we see that under our assumptions $J_{00}=J_{110}=0$ and

$$
\|f\|_{A_{q}^{\gamma}, j}^{q, \text { out }} \leq 2^{q \gamma} \sum_{r \notin D\left(M^{* j}\right)}|r|^{q \gamma}|\widehat{f}(r)|^{q \gamma} .
$$

Using this and taking into account Remark 7 , we obtain (33) and (34). $\diamond$ 


\section{2 $\quad L_{p}$-errors using the best approximation}

In this section, we will show that in some partial cases the results obtained in the previous sections can be sharpened and extended to a wider class of functions $f$ than those considered in Theorems 6 , 9. and 12. Moreover, we show that the $L_{p}$-error estimates for $Q_{j}\left(f, \varphi_{j}, \widetilde{\varphi}_{j}\right)$ can be given using the best approximation.

We restrict ourselves to the case of a diagonal dilation matrix $M=\operatorname{diag}\left(m_{1}, m_{2}, \ldots, m_{d}\right), m_{j} \in \mathbb{Z}$, and the case $\left\{\varphi_{j}\right\}_{j} \in \mathcal{B}$, where $\mathcal{B}=\mathcal{B}_{\delta, R}$ is the class of sequences of trigonometric polynomials introduced in Section 4.1.

We need to specify the class of tempered distributions $\left\{\widetilde{\varphi}_{j}\right\}_{j}$. We will say that a sequence of tempered distributions $\left\{\widetilde{\varphi}_{j}\right\}_{j}$ belongs to the class $\mathcal{S}_{N, p}^{\prime}$ for some $N \geq 0$ and $1 \leq p \leq \infty$ if it satisfies (10) and for any trigonometric polynomial $T_{n}$ such that $\operatorname{spec} T_{n} \subset\left\{k \in \mathbb{Z}^{d}:\left|M^{-n} k\right| \leq 1\right\}$, one has

$$
\left\|T_{n} * \widetilde{\varphi}_{j}\right\|_{p} \leq C_{\widetilde{\varphi}, p} m^{\frac{N}{d}(n-j)}\left\|T_{n}\right\|_{p} \quad \text { for all } \quad n \geq j, \quad j, n \in \mathbb{N} .
$$

Note that in the case of $p=2$ and an isotropic matrix $M$, conditions (10) imply inequality (35). As a simple example of $\left\{\widetilde{\varphi}_{j}\right\}_{j} \in \mathcal{S}_{N, p}^{\prime}$, we can take distributions $\left\{\widetilde{\varphi}_{j}\right\}_{j}$ corresponding to some

differential operator. Namely, let $d=1$ and $\widehat{\varphi_{j}}(l)=\sum_{0 \leq \beta \leq N} c_{\beta}\left(2 \pi i M^{-j} l\right)^{\beta}, N \in \mathbb{Z}_{+}, j \in \mathbb{N}$, then by the well-known Bernstein inequality for trigonometric polynomials $\left\|T_{n}^{(r)}\right\|_{L_{p}(\mathbb{T})} \leq n^{r}\left\|T_{n}\right\|_{L_{p}(\mathbb{T})}$ (see, e.g., [39, p. 215]), we easily derive that $\left\{\widetilde{\varphi}_{j}\right\}_{j} \in \mathcal{S}_{N, p}^{\prime}$.

We will also use the following class of sequences $\mathcal{L}_{p}, 1 \leq p \leq \infty$. We will say that a sequence $\left\{\widetilde{\varphi}_{j}\right\}_{j}$ belongs to $\mathcal{L}_{p}$ if for all $j \in \mathbb{N}$

$$
\left\|\widetilde{\varphi}_{j}\right\|_{\mathcal{L}_{p, j}}:=\left(m^{j} \int_{M^{-j} \mathbb{T}^{d}}\left(\frac{1}{m^{j}} \sum_{k \in D\left(M^{j}\right)}\left|\widetilde{\varphi}_{j}\left(x-M^{-j} k\right)\right|\right)^{p} d x\right)^{\frac{1}{p}}<\infty \quad \text { if } \quad 1 \leq p<\infty
$$

and

$$
\left\|\widetilde{\varphi}_{j}\right\|_{\mathcal{L}_{\infty, j}}:=\frac{1}{m^{j}} \sup _{x \in \mathbb{R}^{d}} \sum_{k \in D\left(M^{j}\right)}\left|\widetilde{\varphi}_{j}\left(x-M^{-j} k\right)\right|<\infty \quad \text { if } \quad p=\infty .
$$

An important example of $\left\{\widetilde{\varphi}_{j}\right\}_{j} \in \mathcal{L}_{p}$ is given by the normalized characteristic functions of $M^{-j}\left[-\frac{1}{2}, \frac{1}{2}\right)^{d}, \widetilde{\varphi}_{j}(x)=m^{j} \chi_{M^{-j}\left[-\frac{1}{2}, \frac{1}{2}\right)^{d}}(x)$. It is worth noting that such $\widetilde{\varphi}_{j}$ provide a periodic counterpart of Kantorovich-Kotelnikov operators studied in [25].

For any $d \times d$-matrix $A$, we denote

$$
\mathcal{T}_{A}:=\left\{T: \operatorname{spec} T \subset\left\{k \in \mathbb{Z}^{d}:\left|A^{-1} k\right|<1\right\}\right\} .
$$

The error of the best approximation of $f \in L_{p}$ by trigonometric polynomials $T \in \mathcal{T}_{A}$ is defined by

$$
E_{A}(f)_{p}:=\inf \left\{\|f-T\|_{p}: T \in \mathcal{T}_{A}\right\} .
$$

We will use the following anisotropic Besov spaces with respect to the matrix $M$. We will say that $f \in \mathbb{B}_{p, q}^{s}(M), 1 \leq p \leq \infty, 0<q \leq \infty$, and $s>0$, if $f \in L_{p}$ and

$$
\|f\|_{\mathbb{B}_{p, q}^{s}(M)}:=\|f\|_{p}+\left(\sum_{\nu=1}^{\infty} m^{\frac{s}{d} q \nu} E_{M^{\nu}}(f)_{p}^{q}\right)^{\frac{1}{q}}<\infty .
$$

For simplicity, we will also denote

$$
\left\|\left\{a_{k}\right\}_{k}\right\|_{\ell_{p, M^{j}}}:= \begin{cases}\left(\frac{1}{m^{j}} \sum_{k \in D\left(M^{j}\right)}\left|a_{k}\right|^{p}\right)^{\frac{1}{p}}, & \text { if } 1 \leq p<\infty, \\ \sup _{k \in D\left(M^{j}\right)}\left|a_{k}\right|, & \text { if } p=\infty .\end{cases}
$$


Lemma 14 Let $1 \leq p \leq \infty$ and $T_{j} \in \mathcal{T}_{\lambda M^{j}}$ for some $\lambda>0$. Then, for any $j \in \mathbb{N}$,

$$
\left\|\left\{T_{j}\left(M^{-j} k\right)\right\}_{k}\right\|_{\ell_{p, M j}} \leq C\left\|T_{j}\right\|_{p}
$$

where the constant $C$ depends only on $p, d$, and $\lambda$.

Proof. In the case $p=\infty$, the proof is obvious. The case $1 \leq p<\infty$ directly follows from the following Marcinkiewicz-Zygmund type inequality (see [29])

$$
\sum_{j=1}^{m}\left|T_{n}\left(\tau_{j}\right)\right|^{p} \leq(p+1) \frac{e}{2}\left(2 n+\frac{1}{\delta}\right) \int_{0}^{1}\left|T_{n}(x)\right|^{p} d x
$$

where $T_{n}$ is a univariate trigonometric polynomial of degree at most $n,-1 / 2 \leq \tau_{1}<\tau_{2}<\cdots<$ $\tau_{m}<1 / 2$, and $\delta=\min \left\{\tau_{2}-\tau_{1}, \tau_{3}-\tau_{2}, \ldots, \tau_{m}-\tau_{m-1}, 1-\left(\tau_{m}-\tau_{1}\right)\right\}$.

Lemma 15 Let $1 \leq p \leq \infty, N \geq 0, \delta \in(0,1 / 2), \widetilde{\varphi} \in \mathcal{S}_{N, p}^{\prime}$, and $f \in \mathbb{B}_{p, 1}^{d / p+N}(M)$. Suppose that the polynomials $T_{\mu}, \mu \in \mathbb{Z}_{+}$, are such that $\operatorname{spec} T_{\mu} \subset D\left(M^{\mu}\right)$ and

$$
\left\|f-T_{\mu}\right\|_{p} \leq \sigma E_{\delta M^{\mu}}(f)_{p}
$$

with some constant $\sigma$ independent of $f$ and $\mu$. Then the sequence $\left\{\left\langle T_{\mu}, \widetilde{\varphi}_{j}\left(\cdot-M^{-j} k\right)\right\rangle\right\}_{\mu=0}^{\infty}$ converges uniformly with respect to $k \in \mathbb{Z}^{d}$ and $j \in \mathbb{N}$, and the limit does not depend on the choice of polynomials $T_{\mu}$. Moreover, for all $n \in \mathbb{N}$,

$$
\sum_{\mu=n}^{\infty}\left\|\left\{\left\langle T_{\mu+1}-T_{\mu}, \widetilde{\varphi}_{j}\left(\cdot-M^{-j} k\right)\right\rangle\right\}_{k}\right\|_{\ell_{p, M^{j}}} \leq C m^{-\left(\frac{1}{p}+\frac{N}{d}\right) j} \sum_{\mu=n}^{\infty} m^{\left(\frac{1}{p}+\frac{N}{d}\right) \mu} E_{\delta M^{\mu}}(f)_{p},
$$

where the constant $C$ depends only on $d, p, M$, and $\sigma$.

Proof. Let $n \geq j, n \in \mathbb{N}$. Using Lemma 14, condition 35, we obtain

$$
\begin{aligned}
& \sum_{\mu=n}^{\infty}\left\|\left\{\left\langle T_{\mu+1}, \widetilde{\varphi}_{j}\left(\cdot-M^{-j} k\right)\right\rangle-\left\langle T_{\mu}, \widetilde{\varphi}_{j}\left(\cdot-M^{-j} k\right)\right\rangle\right\}_{k}\right\|_{\ell_{p, M^{j}}} \\
& =\sum_{\mu=n}^{\infty}\left\|\left\{\left\langle T_{\mu+1}-T_{\mu}, \widetilde{\varphi}_{j}\left(\cdot-M^{-j} k\right)\right\rangle\right\}_{k}\right\|_{\ell_{p, M^{j}}}=\sum_{\mu=n}^{\infty}\left\|\left\{\left(T_{\mu+1}^{-}-T_{\mu}^{-}\right) * \widetilde{\varphi}_{j}\left(M^{-j} k\right)\right\}_{k}\right\|_{\ell_{p, M^{j}}} \\
& \leq m^{-\frac{j}{p}} \sum_{\mu=n}^{\infty} m^{\frac{\mu}{p}}\left\|\left\{\left(T_{\mu+1}^{-}-T_{\mu}^{-}\right) * \widetilde{\varphi}_{j}\left(M^{-\mu} k\right)\right\}_{k}\right\|_{\ell_{p, M} \mu} \leq C_{1} m^{-\frac{j}{p}} \sum_{\mu=n}^{\infty} m^{\frac{\mu}{p}}\left\|\left(T_{\mu+1}^{-}-T_{\mu}^{-}\right) * \widetilde{\varphi}_{j}\right\|_{p} \\
& \leq C_{2} m^{-\left(\frac{1}{p}+\frac{N}{d}\right) j} \sum_{\mu=n}^{\infty} m^{\left(\frac{1}{p}+\frac{N}{d}\right) \mu}\left\|T_{\mu+1}^{-}-T_{\mu}^{-}\right\|_{p} \leq C_{2} m^{-\left(\frac{1}{p}+\frac{N}{d}\right) j} \sum_{\mu=n}^{\infty} m^{\left(\frac{1}{p}+\frac{N}{d}\right) \mu} E_{\delta M^{\mu}}(f)_{p} .
\end{aligned}
$$

The latter series is convergent since $f \in \mathbb{B}_{p, 1}^{d / p+N}(M)$, which yields that the sequence $\left\{\left\langle T_{\mu}, \widetilde{\varphi}_{j}(\cdot-\right.\right.$ $\left.\left.\left.M^{-j} k\right)\right\rangle\right\}_{\mu=1}^{\infty}$ is convergent for every $k \in \mathbb{Z}^{d}$ and $j \in \mathbb{N}$. By analogy with (36), it is also easy to check that the limit does not depend on the choice of functions $T_{\mu}$. $\diamond$

In the previous section, the operator

$$
Q_{j}\left(f, \varphi_{j}, \widetilde{\varphi}_{j}\right)=\frac{1}{m^{j}} \sum_{k \in D\left(M^{j}\right)}\left\langle f, \widetilde{\varphi}_{j}\left(\cdot-M^{-j} k\right)\right\rangle \varphi_{j}\left(\cdot-M^{-j} k\right),
$$


was considered for functions $f$ whose Fourier coefficients decay sufficiently fast. In particular, supposing that (10) holds and $f \in A_{q}^{\gamma}$ with $\gamma \geq N$, we defined the inner product by

$$
\left\langle f, \widetilde{\varphi}_{j}\left(\cdot-M^{-j} k\right)\right\rangle=\sum_{l \in \mathbb{Z}^{d}} \widehat{f}(l) \overline{\widehat{\varphi}_{j}(l)} e^{2 \pi i\left(k, M^{*-j} l\right)} .
$$

Taking into account Lemma 15 and using condition (35), we extend the functional $\left\langle f, \widetilde{\varphi}_{j}\left(\cdot-M^{-j} k\right)\right\rangle$ from $f \in A_{q}^{\gamma}$ to the Besov spaces $\mathbb{B}_{p, 1}^{d / p+N}(M)$ as follows.

Definition 16 Let $1 \leq p \leq \infty, N \geq 0, \widetilde{\varphi} \in S_{N, p}^{\prime}$, and the polynomials $T_{\mu}$ be as in Lemma 15. For every $f \in \mathbb{B}_{p, 1}^{d / p+N}(M)$ and $k \in \mathbb{Z}^{d}$, we set

$$
\left\langle f, \widetilde{\varphi}_{j}\left(\cdot-M^{-j} k\right)\right\rangle:=\lim _{\mu \rightarrow \infty}\left\langle T_{\mu}, \widetilde{\varphi}_{j}\left(\cdot-M^{-j} k\right)\right\rangle, \quad j \in \mathbb{N} .
$$

Now, if $\widetilde{\varphi} \in S_{N, p}^{\prime}$, then the quasi-projection operators

$$
Q_{j}(f, \varphi, \widetilde{\varphi})=\sum_{k \in \mathbb{Z}^{d}}\left\langle f, \widetilde{\varphi}_{j}\left(\cdot-M^{-j} k\right)\right\rangle \varphi_{j}\left(\cdot-M^{-j} k\right)
$$

are defined on the space $\mathbb{B}_{p, 1}^{d / p+N}(M)$ for a wide class of appropriate functions $\varphi$.

Note that below we will consider the operators $Q_{j}(f, \varphi, \widetilde{\varphi})$ for the sequences $\left\{\widetilde{\varphi}_{j}\right\}_{j}$ belonging to $\mathcal{L}_{q}$. In this case, the inner product $\left\langle f, \widetilde{\varphi}_{j}\left(\cdot-M^{-j} k\right)\right\rangle$ has sense for any $f \in L_{p}, 1 / p+1 / q=1$. As usual, we have $\left\langle f, \widetilde{\varphi}_{j}\left(\cdot-M^{-j} k\right)\right\rangle=\int_{\mathbb{T}^{d}} f(t) \widetilde{\widetilde{\varphi}_{j}}\left(t-M^{-j} k\right) d t$.

In what follows, a Fourier multiplier operator associated with a function $\varphi_{j}$ is denoted by $S_{\varphi_{j}}$, i.e. for any function $f \in L_{p}, 1 \leq p \leq \infty$, we set

$$
S_{\varphi_{j}}(f ; x):=\sum_{k \in \mathbb{Z}^{d}} \widehat{\varphi_{j}}(k) \widehat{f}(k) e^{2 \pi i(k, x)}
$$

Denote also

$$
K_{\varphi_{j}, q}:=\sup _{\|f\|_{q} \leq 1}\left\|S_{\varphi_{j}}(f ; \cdot)\right\|_{q} .
$$

The standard example of such operators is the partial sums of Fourier series. For example, if $\widehat{\varphi_{j}}(\xi)=\chi_{M^{j}\left[-\frac{1}{2}, \frac{1}{2}\right)^{d}}(\xi)$, then $S_{\varphi_{j}}$ represents the rectangular partial sums of Fourier series and

$$
K_{\varphi_{j}, q} \asymp \begin{cases}1, & 1<q<\infty \\ j^{d}, & q=1 \text { or } \infty .\end{cases}
$$

We will need the following two auxiliary lemmas.

Lemma 17 Let $1 \leq p \leq \infty, 1 / p+1 / q=1,\left\{a_{k}\right\}_{k \in D\left(M^{j}\right)} \in \mathbb{C}$, and $\left\{\varphi_{j}\right\}_{j} \in \mathcal{B}$. Then, for any $j \in \mathbb{N}$,

$$
\left\|\frac{1}{m^{j}} \sum_{k \in D\left(M^{j}\right)} a_{k} \varphi_{j}\left(\cdot-M^{-j} k\right)\right\|_{p} \leq C K_{\varphi_{j}, q}\left\|\left\{a_{k}\right\}_{k}\right\|_{\ell_{p, M^{j}}} .
$$

where the constant $C$ does not depend on $j$ and $\left\{a_{k}\right\}$.

Proof. Consider the case $1 \leq p<\infty$. By duality, we can find a function $g_{j}$ such that $\left\|g_{j}\right\|_{q} \leq 1$ and

$$
\begin{aligned}
\left\|\sum_{k \in D\left(M^{j}\right)} a_{k} \varphi_{j}\left(\cdot-M^{-j} k\right)\right\|_{p} & =\left|\left\langle\sum_{k \in D\left(M^{j}\right)} a_{k} \varphi_{j}\left(\cdot-M^{-j} k\right), g_{j}\right\rangle\right| \\
& =\left|\sum_{k \in D\left(M^{j}\right)} a_{k}\left\langle\varphi_{j}\left(\cdot-M^{-j} k\right), g_{j}\right\rangle\right| .
\end{aligned}
$$


Applying Hölder's inequality and Lemma [14, taking into account that $\left\langle\varphi_{j}\left(\cdot-M^{-j} k\right), g_{j}\right\rangle=\varphi_{j} *$ $g_{j}{ }^{-}\left(-M^{-j} k\right)$ and $\varphi_{j} * g_{j}^{-} \in \mathcal{T}_{R M^{j}}$, we obtain

$$
\begin{aligned}
& \sum_{k \in D\left(M^{j}\right)}\left|a_{k}\left\langle\varphi_{j}\left(\cdot-M^{-j} k\right), g_{j}\right\rangle\right| \leq\left(\sum_{k \in D\left(M^{j}\right)}\left|a_{k}\right|^{p}\right)^{\frac{1}{p}}\left(\sum_{k \in D\left(M^{j}\right)}\left|\left\langle\varphi_{j}\left(\cdot-M^{-j} k\right), g_{j}\right\rangle\right|^{q}\right)^{\frac{1}{q}} \\
& \quad \leq C\left(\sum_{k \in D\left(M^{j}\right)}\left|a_{k}\right|^{p}\right)^{\frac{1}{p}} m^{j / q}\left\|\varphi_{j} * g_{j}^{-}\right\|_{q} \leq C\left(\sum_{k \in D\left(M^{j}\right)}\left|a_{k}\right|^{p}\right)^{\frac{1}{p}} m^{j / q} K_{\varphi_{j}, q}\left\|g_{j}\right\|_{q} \\
& \quad \leq C m^{j} K_{\varphi_{j}, q}\left\|\left\{a_{k}\right\}_{k}\right\|_{\ell_{p, M^{j}}} .
\end{aligned}
$$

Then, combining (38) and (39), we prove the lemma for $p \neq \infty$. In the case $p=\infty$, the proof is similar.

Lemma 18 Let $1 \leq p \leq \infty, 1 / p+1 / q=1$, and $\left\{\widetilde{\varphi}_{j}\right\}_{j} \in \mathcal{L}_{q}$. Then, for any $f \in L_{p}$ and $j \in \mathbb{N}$, we have

$$
\left\|\left\{\left\langle f, \widetilde{\varphi}_{j}\left(\cdot-M^{-j} k\right)\right\rangle\right\}_{k}\right\|_{\ell_{p, M}} \leq\left\|\widetilde{\varphi}_{j}\right\|_{\mathcal{L}_{q, j}}\|f\|_{p}
$$

Proof. In the case $p=\infty$, the proof is obvious since $\left\|\widetilde{\varphi}_{j}\right\|_{\mathcal{L}_{1, j}}=\left\|\widetilde{\varphi}_{j}\right\|_{1}$. For $p<\infty$, we have

$$
\begin{aligned}
\left(\sum_{k \in D\left(M^{j}\right)}\left|\left\langle f, \widetilde{\varphi}_{j}\left(\cdot-M^{-j} k\right)\right\rangle\right|^{p}\right)^{\frac{1}{p}} & \left(\sum_{k \in D\left(M^{j}\right)}\left|\sum_{l \in D\left(M^{j}\right)} \int_{\frac{l_{1}}{m_{1}^{j}}}^{\frac{l_{1}+1}{m_{1}^{j}}} d x_{1} \cdots \int_{\frac{l_{d}}{m_{d}^{j}}}^{\frac{l_{d}+1}{m_{d}^{j}}} f(x) \overline{\widetilde{\varphi}_{j}}\left(x-M^{-j} k\right) d x_{d}\right|^{p}\right)^{\frac{1}{p}} \\
& =\left(\sum_{k \in D\left(M^{j}\right)}\left|\int_{M^{-j} \mathbb{T}^{d}} \sum_{l \in D\left(M^{j}\right)} f\left(x+M^{-j} l\right) \overline{\widetilde{\varphi}_{j}}\left(x-M^{-j}(k-l)\right) d x\right|^{p}\right)^{\frac{1}{p}} \\
& \leq \int_{M^{-j} \mathbb{T}^{d}}\left(\sum_{k \in D\left(M^{j}\right)}\left|\sum_{l \in D\left(M^{j}\right)} f\left(x+M^{-j} l\right) \overline{\widetilde{\varphi}_{j}}\left(x-M^{-j}(k-l)\right)\right|^{p}\right)^{\frac{1}{p}} d x
\end{aligned}
$$

where the last formula follows from Minkowski's inequality.

Next, applying Young's inequality for the discrete convolution and Hölder's inequality, we derive that the last expression can be estimated from above by

$$
\begin{aligned}
& \int_{M^{-j} \mathbb{T}^{d}}\left(\sum_{l \in D\left(M^{j}\right)}\left|f\left(x+M^{-j} l\right)\right|^{p}\right)^{\frac{1}{p}} \sum_{k \in D\left(M^{j}\right)}\left|\widetilde{\varphi}_{j}\left(x-M^{-j} k\right)\right| d x \\
& \leq\|f\|_{p}\left(\int_{M^{-j} \mathbb{T}^{d}}\left(\sum_{k \in D\left(M^{j}\right)}\left|\widetilde{\varphi}_{j}\left(x-M^{-j} k\right)\right|\right)^{q} d x\right)^{\frac{1}{q}}=m^{j / p}\left\|\widetilde{\varphi}_{j}\right\|_{\mathcal{L}_{q, j}}\|f\|_{p},
\end{aligned}
$$

which proves the lemma. $\diamond$

Now, we are ready to formulate and prove the main results of this section. We start from the case of strictly compatible functions/distributions $\varphi_{j}$ and $\widetilde{\varphi}_{j}$. 
Theorem 19 Let $1 \leq p \leq \infty, 1 / p+1 / q=1$, and $N \geq 0$. Suppose that $\left\{\widetilde{\varphi}_{j}\right\}_{j} \in \mathcal{S}_{N, p}^{\prime},\left\{\varphi_{j}\right\}_{j} \in \mathcal{B}$ with respect to the parameter $\delta \in(0,1 / 2)$, and $\varphi_{j}$ and $\widetilde{\varphi}_{j}$ are strictly compatible with respect to $\delta$. Then, for any $f \in \mathbb{B}_{p, 1}^{d / p+N}(M)$ and $j \in \mathbb{N}$, we have

$$
\left\|f-Q_{j}\left(f, \varphi_{j}, \widetilde{\varphi}_{j}\right)\right\|_{p} \leq C K_{\varphi_{j}, q} m^{-j\left(\frac{1}{p}+\frac{N}{d}\right)} \sum_{\nu=j}^{\infty} m^{\left(\frac{1}{p}+\frac{N}{d}\right) \nu} E_{\delta M^{\nu}}(f)_{p},
$$

if, additionally, $\left\{\widetilde{\varphi}_{j}\right\}_{j} \in \mathcal{L}_{q}$, then for any $f \in L_{p}$, we have

$$
\left\|f-Q_{j}\left(f, \varphi_{j}, \widetilde{\varphi}_{j}\right)\right\|_{p} \leq C K_{\varphi_{j}, q}\left\|\widetilde{\varphi}_{j}\right\|_{\mathcal{L}_{q, j}} E_{\delta M^{j}}(f)_{p},
$$

where the constant $C$ does not depend on $f$ and $j$.

Proof. Let $T_{j} \in \mathcal{T}_{\delta M^{j}}$ be such that $\left\|f-T_{j}\right\|_{p}=E_{\delta M^{j}}(f)_{p}$, then

$$
\begin{aligned}
\left\|f-\frac{1}{m^{j}} \sum_{k \in D\left(M^{j}\right)}\left\langle f, \widetilde{\varphi}_{j}\left(\cdot-M^{-j} k\right)\right\rangle \varphi_{j}\left(\cdot-M^{-j} k\right)\right\|_{p} \\
\leq\left\|f-T_{j}\right\|_{p}+\left\|T_{j}-\frac{1}{m^{j}} \sum_{k \in D\left(M^{j}\right)}\left\langle T_{j}, \widetilde{\varphi}_{j}\left(\cdot-M^{-j} k\right)\right\rangle \varphi_{j}\left(\cdot-M^{-j} k\right)\right\|_{p} \\
\quad+\left\|\frac{1}{m^{j}} \sum_{k \in D\left(M^{j}\right)}\left\langle f-T_{j}, \widetilde{\varphi}_{j}\left(\cdot-M^{-j} k\right)\right\rangle \varphi_{j}\left(\cdot-M^{-j} k\right)\right\|_{p}:=I_{1}+I_{2}+I_{3} .
\end{aligned}
$$

To estimate $I_{2}$, we note that by Theorem 12 ,

$$
T_{j}(x)-\frac{1}{m^{j}} \sum_{k \in D\left(M^{j}\right)}\left\langle T_{j}, \widetilde{\varphi}_{j}\left(\cdot-M^{-j} k\right)\right\rangle \varphi_{j}\left(x-M^{-j} k\right)=0
$$

which implies that

$$
I_{2}=0
$$

Consider $I_{3}$. Using Lemmas 17 and 15, we derive

$$
\begin{aligned}
I_{3} & \leq C_{1} K_{\varphi_{j}, q}\left\|\left\{\left\langle f-T_{j}, \widetilde{\varphi}_{j}\left(\cdot-M^{-j} k\right)\right\rangle\right\}_{k}\right\|_{\ell_{p, M^{j}}} \\
& \leq C_{2} K_{\varphi_{j}, q} \sum_{\mu=n}^{\infty}\left\|\left\{\left\langle T_{\mu+1}-T_{\mu}, \widetilde{\varphi}_{j}\left(\cdot-M^{-j} k\right)\right\rangle\right\}_{k}\right\|_{\ell_{p, M} j} \leq C_{3} K_{\varphi_{j}, q} \sum_{\mu=n}^{\infty} m^{\mu\left(\frac{N}{d}+\frac{1}{p}\right)} E_{\delta M^{\mu}}(f)_{p} .
\end{aligned}
$$

Then, combining (42), (43), and (44), we prove (40).

To obtain inequality (41), it is sufficient to use inequalities (42) and (43) as well as the following estimate

$$
I_{3} \leq C_{4} K_{\varphi_{j}, q}\left\|\left\{\left\langle f-T_{j}, \widetilde{\varphi}_{j}\left(\cdot-M^{-j} k\right)\right\rangle\right\}_{k}\right\|_{\ell_{p, M^{j}}} \leq C_{5} K_{\varphi_{j}, q}\left\|\widetilde{\varphi}_{j}\right\|_{\mathcal{L}_{q, j}}\left\|f-T_{j}\right\|_{p},
$$

which easily follows from Lemmas 17 and 18, $\diamond$

Applying Hausdorff-Young's inequality (21) to the right-hand sides of (40) and (41), we derive the following improvements of the error estimate given in Theorem 12

Corollary 20 Let $2 \leq p \leq \infty, 1 / p+1 / q=1$, and $N \geq 0$. Suppose that $M=\lambda I_{d}, \lambda>1$, $\left\{\widetilde{\varphi}_{j}\right\}_{j} \in \mathcal{S}_{N, p}^{\prime},\left\{\varphi_{j}\right\}_{j} \in \mathcal{B}$ with respect to the parameter $\delta \in(0,1 / 2)$, and $\varphi_{j}$ and $\widetilde{\varphi}_{j}$ are strictly compatible with respect to $\delta$. Then, for any $f \in \mathbb{B}_{p, 1}^{d / p+N}(M)$ and $j \in \mathbb{N}$, we have

$$
\left\|f-Q_{j}\left(f, \varphi_{j}, \widetilde{\varphi}_{j}\right)\right\|_{p} \leq C K_{\varphi_{j}, q} \lambda^{-j\left(\frac{d}{p}+N\right)} \sum_{\nu=j}^{\infty} \lambda^{\left(\frac{d}{p}+N\right) \nu}\left(\sum_{|r| \geq \delta \lambda^{\nu}}|\widehat{f}(r)|^{q}\right)^{1 / q}
$$


if, additionally, $\left\{\widetilde{\varphi}_{j}\right\}_{j} \in \mathcal{L}_{q}$, then

$$
\left\|f-Q_{j}\left(f, \varphi_{j}, \widetilde{\varphi}_{j}\right)\right\|_{p} \leq C K_{\varphi_{j}, q}\left\|\widetilde{\varphi}_{j}\right\|_{\mathcal{L}_{q, j}}\left(\sum_{|r| \geq \delta \lambda^{j}}|\widehat{f}(r)|^{q}\right)^{1 / q}
$$

where the constant $C$ does not depend on $f$ and $j$.

In light of Proposition 13, it is not difficult to derive the following improvements of Theorem 19. in which we replace the best approximation $E_{\delta M^{j}}(f)_{p}$ by

$$
E_{M^{j}}^{*}(f)_{p}:=\inf \left\{\|f-T\|_{p}: \operatorname{spec} T \subset D\left(M^{j}\right)\right\}, \quad j \in \mathbb{N} .
$$

Proposition 21 If under the assumptions of Theorem 19, equality (28) holds for all $l \in D\left(M^{j}\right)$ and $\operatorname{spec} \varphi_{j} \subset D\left(M^{j}\right)$, then, for any $f \in \mathbb{B}_{p, 1}^{d / p+N}(M)$ and $j \in \mathbb{N}$, we have

$$
\left\|f-Q_{j}\left(f, \varphi_{j}, \widetilde{\varphi}_{j}\right)\right\|_{p} \leq C K_{\varphi_{j}, q} m^{-j\left(\frac{1}{p}+\frac{N}{d}\right)} \sum_{\nu=j}^{\infty} m^{\left(\frac{1}{p}+\frac{N}{d}\right) \nu} E_{M^{\nu}}^{*}(f)_{p}
$$

if, additionally, $\left\{\widetilde{\varphi}_{j}\right\}_{j} \in \mathcal{L}_{q}$, then for any $f \in L_{p}$, we have

$$
\left\|f-Q_{j}\left(f, \varphi_{j}, \widetilde{\varphi}_{j}\right)\right\|_{p} \leq C K_{\varphi_{j}, q}\left\|\widetilde{\varphi}_{j}\right\|_{\mathcal{L}_{q, j}} E_{M^{j}}^{*}(f)_{p},
$$

where the constant $C$ does not depend on $f$ and $j$.

Now, we consider the case of weakly compatible functions/distributions $\varphi_{j}$ and $\widetilde{\varphi}_{j}$.

Theorem 22 Let $2 \leq p \leq \infty, 1 / p+1 / q=1$, and $N \geq 0$. Suppose that $\left\{\widetilde{\varphi}_{j}\right\}_{j} \in \mathcal{S}_{N, p}^{\prime},\left\{\varphi_{j}\right\}_{j} \in \mathcal{B}$ with respect to the parameter $\delta \in(0,1 / 2)$, and $\varphi_{j}$ and $\widetilde{\varphi}_{j}$ are weakly compatible of order $s>0$. Then, for any $f \in \mathbb{B}_{p, 1}^{d / p+N}(M)$ and $j \in \mathbb{N}$, we have

$$
\left\|f-Q_{j}\left(f, \varphi_{j}, \widetilde{\varphi}_{j}\right)\right\|_{p} \leq C\left(\left\|M^{-j}\right\|^{s}\|f\|_{A_{q}^{s}, j}^{I n}+K_{\varphi_{j}, q} m^{-j\left(\frac{1}{p}+\frac{N}{d}\right)} \sum_{\nu=j}^{\infty} m^{\left(\frac{1}{p}+\frac{N}{d}\right) \nu} E_{\delta M^{\nu}}(f)_{p}\right)
$$

if, additionally, $\left\{\widetilde{\varphi}_{j}\right\}_{j} \in \mathcal{L}_{q}$, then for any $f \in L_{p}$, we have

$$
\left\|f-Q_{j}\left(f, \varphi_{j}, \widetilde{\varphi}_{j}\right)\right\|_{p} \leq C\left(\left\|M^{-j}\right\|^{s}\|f\|_{A^{s}, j}^{I n}+K_{\varphi_{j}, q}\left\|\widetilde{\varphi}_{j}\right\|_{\mathcal{L}_{q, j}} E_{\delta M^{j}}(f)_{p}\right),
$$

where the constant $C$ does not depend on $f$ and $j$. by

Proof. First, we prove inequality (46). Consider the de la Vallée-Poussin means $V_{j}(f)$ defined

$$
V_{j}(f)(x)=\sum_{k \in \mathbb{Z}^{d}} v_{\delta}\left(M^{-j} k\right) \widehat{f}(k) e^{2 \pi i(k, x)},
$$

where $v_{\delta} \in C^{\infty}\left(\mathbb{R}^{d}\right), v_{\delta}(\xi)=1$ if $|\xi|<\delta$ and $v_{\delta}(\xi)=0$ if $\xi \notin\left(-\frac{1}{2}, \frac{1}{2}\right)^{d}$. As usual, we have for any $j \in \mathbb{N}$ that

$$
\left\|f-V_{j}(f)\right\|_{p} \leq\left(1+\left\|V_{j}\right\|_{1}\right) E_{\delta M^{j}}(f)_{p} \leq c E_{\delta M^{j}}(f)_{p},
$$

where the constant $c$ does not depend on $j$ and $f$. 
Repeating the proof of Theorem 19 with $V_{j}(f)$ instead of the polynomials of the best approximation $T_{j}$ and using (48), we derive

$$
\begin{aligned}
& \left\|f-\frac{1}{m^{j}} \sum_{k \in D\left(M^{j}\right)}\left\langle f, \widetilde{\varphi}_{j}\left(\cdot-M^{-j} k\right)\right\rangle \varphi_{j}\left(\cdot-M^{-j} k\right)\right\|_{p} \\
& \leq\left\|V_{j}(f)-\frac{1}{m^{j}} \sum_{k \in D\left(M^{j}\right)}\left\langle V_{j}(f), \widetilde{\varphi}_{j}\left(\cdot-M^{-j} k\right)\right\rangle \varphi_{j}\left(\cdot-M^{-j} k\right)\right\|_{p} \\
& \quad+C_{1} K_{\varphi_{j}, q} m^{-j\left(\frac{1}{p}+\frac{N}{d}\right)} \sum_{\nu=j}^{\infty} m^{\left(\frac{1}{p}+\frac{N}{d}\right) \nu} E_{\delta M^{\nu}}(f)_{p}:=J_{1}+J_{2} .
\end{aligned}
$$

By Theorem 9, taking into account that the Strang-Fix conditions of order $s$ for $\varphi_{j}$ and the weak compatibility conditions for $\varphi_{j}$ and $\widetilde{\varphi}_{j}$ of order $s$ are satisfied (see the proof of Theorem 12), we derive

$$
J_{1} \leq C_{2}\left\|M^{-j}\right\|^{s}\left\|V_{j}(f)\right\|_{A_{q}^{s}, j}^{I n} \leq C_{3}\left\|M^{-j}\right\|^{s}\|f\|_{A_{q}^{s}, j}^{I n} .
$$

Thus, combining (49) and (50), we prove (46).

The proof of estimate (47) is similar. One needs only to use inequality (45) instead of (44).

Next, using the Hausdorff-Young inequality, we obtain the following corollaries, which provide two shaper versions of Theorem 9

Corollary 23 If under the assumptions of Theorem [2., $\left\{\widetilde{\varphi}_{j}\right\}_{j} \in \mathcal{L}_{q}$ and $f \in A_{q}^{\gamma}$, where $\gamma>0$, then

$$
\left\|f-Q_{j}\left(f, \varphi_{j}, \widetilde{\varphi}_{j}\right)\right\|_{p} \leq C K_{\varphi_{j}, q}\left\|M^{-j}\right\|^{\min \{s, \gamma\}}\|f\|_{A_{q}^{\gamma}} .
$$

In particular, if $M=\lambda I_{d}$, $\sup _{j} K_{\varphi_{j}, q}<\infty$, and $\widehat{f}(n)=\mathcal{O}\left(|n|^{-\kappa}\right)$ for some $\kappa>d / q$, then

$$
\left\|f-Q_{j}\left(f, \varphi_{j}, \widetilde{\varphi}_{j}\right)\right\|_{p}=\mathcal{O}\left(\lambda^{-j \min \{s, \kappa-d / q\}}\right) .
$$

Corollary 24 If under the assumptions of Theorem 22, $M=\lambda I_{d}, 0<s \leq d / p+N, f \in A_{q}^{s} \cap$ $\mathbb{B}_{p, 1}^{d / p+N}(M)$, then

$$
\left\|f-Q_{j}\left(f, \varphi_{j}, \widetilde{\varphi}_{j}\right)\right\|_{p} \leq C K_{\varphi_{j}, q} \lambda^{-j s} .
$$

Remark 25 Note that this result provides a shaper version of Theorem 9 because there exist functions $f \notin A_{q}^{\gamma}, \gamma>d / p+N$, for which conditions of Corollary 24 are valid. As an example in the case $d=1$, one can take the function

$$
f(x)=\sum_{k=1}^{\infty} \frac{e^{i k \log k}}{k^{\frac{1}{2}+\frac{1}{p}+N+\varepsilon}} e^{2 \pi i k x}, \quad \varepsilon>0 .
$$

It follows from 44. Ch. V, Theorem 4.2] that $f^{(N)} \in \operatorname{Lip}\left(\frac{1}{p}+\varepsilon\right)$ and, therefore, by the classical Jackson inequality (see, e.g., [39, p. 260]), we have that $E_{\lambda^{\nu}}(f)_{p}=\mathcal{O}\left(\lambda^{-\gamma \nu}\right)$ with $\gamma=1 / p+N+\varepsilon$, which implies that $f \in B_{p, 1}^{1 / p+N}$. At the same time, $f \in A_{q}^{s}$ for $s<\gamma-1 / q$ and $f \notin A_{q}^{\gamma}$.

\section{$5 \quad$ Examples}

1. In fact, for a sequence of periodic distributions $\left\{\widetilde{\varphi}_{j}\right\}$ satisfying conditions (10) and such that $\left|\widehat{\widetilde{\varphi}_{j}}(k)\right| \geq c>0$ for $k \in \mathbb{Z}^{d}:\left|M^{*-j} k\right| \leq \delta$ for any $j \in \mathbb{N}$ and some fixed $\delta \in\left(0, \frac{1}{2}\right)$, an appropriate 
strictly compatible sequence of trigonometric polynomials $\varphi_{j}$ can be constructed via defining its Fourier coefficients by condition (28), namely

$$
\overline{\widehat{\varphi_{j}}(k)}=\frac{1}{\widehat{\widehat{\varphi}_{j}}(k)}, \quad k \in \mathbb{Z}^{d}:\left|M^{*-j} k\right| \leq \delta
$$

and $\widehat{\varphi_{j}}(k)=0$ for others $k \in \mathbb{Z}^{d}$. Obviously, the sequence $\left\{\varphi_{j}\right\}_{j}$ belongs to $\mathcal{B}$, and hence we are under the assumptions of Theorem 12 or Theorem 19 (if $\left\{\widetilde{\varphi}_{j}\right\}_{j} \in \mathcal{S}_{N, p}^{\prime}$ and $M$ is diagonal). For instance, assume that $\widetilde{\varphi}_{j}$ is a periodic distribution corresponding to some differential operator. Namely, let

$$
\widehat{\widetilde{\varphi}_{j}}(k)=\sum_{[\beta] \leq N} c_{\beta}\left(2 \pi i M^{*-j} k\right)^{\beta}, \quad N \in \mathbb{Z}_{+}, \quad c_{\mathbf{0}} \neq 0 .
$$

For any good enough function $f$, we have

$$
\begin{aligned}
\left\langle f, \widetilde{\varphi}_{j}\left(\cdot-M^{-j} k\right)\right\rangle & =\sum_{l \in \mathbb{Z}^{d}} \widehat{f}(l) \widehat{\widehat{\bar{\varphi}_{j}}(l)} e^{2 \pi i\left(M^{-j} k, l\right)}=\sum_{[\beta] \leq N} \bar{c}_{\beta} \sum_{l \in \mathbb{Z}^{d}} \widehat{f}(l)\left(-2 \pi i M^{*-j} l\right)^{\beta} e^{2 \pi i\left(k, M^{*-j} l\right)} \\
& =\sum_{[\beta] \leq N} \bar{c}_{\beta}\left[D^{\beta} f\left(-M^{-j} \cdot\right)\right](k)=:\left[L f\left(M^{-j} \cdot\right)\right](k) .
\end{aligned}
$$

This sequence of periodic distributions $\widetilde{\varphi}_{j}$ satisfies conditions (10). Since $\widehat{\widetilde{\varphi}_{j}}(k)=P\left(M^{*-j} k\right)$, where $P$ is an algebraic polynomial and $P(\mathbf{0}) \neq 0$, there exists $\delta>0$ such that $\left|{\widetilde{\varphi_{j}}}(k)\right| \geq c>0$ whenever $k \in \mathbb{Z}^{d}:\left|M^{*-j} k\right| \leq \delta$. In particular, if $N=0$ and $c_{\mathbf{0}}=1$, then $\widehat{\bar{\varphi}_{j}}(k)=1$ for $k \in \mathbb{Z}^{d}$. For the strict compatibility, one can take $\widehat{\varphi_{j}}(k)=1$ for $k \in \mathbb{Z}^{d}:\left|M^{*-j} k\right| \leq \frac{1}{2}$ and $\widehat{\varphi_{j}}(k)=0$ for other $k \in \mathbb{Z}^{d}$, i.e., $\left\{\varphi_{j}\right\}_{j}$ is a sequence of Dirichlet-type kernels.

2. Let $\widehat{\varphi_{j}}(k)=1$ for $k \in \mathbb{Z}^{d}$. In order to achieve only the weak compatibility, we can take truncated Fejer-type kernels $\varphi_{j}$ defined by

$$
\widehat{\varphi_{j}}(k)= \begin{cases}1-C_{F}\left\|M^{*-j} k\right\|_{\infty}, & \text { if }\left|M^{*-j} k\right| \leq \delta, \\ 0, & \text { otherwise }\end{cases}
$$

where $\delta \in\left(0, \frac{1}{2}\right)$ and $C_{F}$ is a positive real number. In this case

$$
1-\widehat{\widetilde{\varphi}_{j}}(k) \widehat{\varphi_{j}}(k)=C_{F}\left\|M^{*-j} k\right\|_{\infty} \leq C_{F}\left|M^{-j} k\right|, \quad k \in D\left(M^{* j}\right),
$$

which means that compatibility condition (12) is valid for $s=1$. Thus, we are in case of Theorem 22.

Alongside, consider the following Fejer-type kernels defined by

$$
\widehat{\varphi_{j}}(k)= \begin{cases}1-C_{F}\left\|M^{*-j} k\right\|_{\infty}, & \text { if }\left\|M^{*-j} k\right\|_{\infty} \leq 1, \\ 0, & \text { otherwise. }\end{cases}
$$

In this case, we have to check the Strang-Fix conditions only for points $M^{* j} n+r$, where $\|n\|_{\infty}=1$ and $r \in D\left(M^{* j}\right)$. Then

$$
\left|\widehat{\varphi_{j}}\left(M^{* j} n+r\right)\right|=\left|1-\left\|n+M^{*-j} r\right\|_{\infty}\right|=\left|\|n\|_{\infty}-\left\|n+M^{*-j} r\right\|_{\infty}\right| \leq\left\|M^{*-j} r\right\|_{\infty} \leq\left|M^{-j} r\right| .
$$

Therefore, the Strang-Fix condition of order 1 for the sequence $\left\{\varphi_{j}\right\}_{j}$ is satisfied. So, we are under assumptions of Theorem [6 or Theorem 9 with $s=1$.

3. Next, we discuss sequences $\left\{\varphi_{j}\right\}_{j}$ obtained by periodization of splines, which are applicable in Theorem 6 or 9 . We restrict ourselves to the case $d=1$, and $M$ is an integer greater than 1 . Assume that $\varphi^{s}$ is a B-spline of an even order $s$, whose Fourier transform is given by

$$
\widehat{\varphi^{s}}(\xi)=\left(\frac{\sin \pi \xi}{\pi \xi}\right)^{s} .
$$


Recall that $\operatorname{supp} \varphi^{s} \subset[-s / 2, s / 2]$ and the non-periodic Strang-Fix conditions of order $s$ are valid for $\varphi^{s}$. Therefore, after periodization of $\varphi^{s}$ by (9), we obtain the functions $\varphi_{j}^{s}(x)$ whose Fourier coefficients are

$$
\widehat{\varphi_{j}^{s}}(k)=\left(\frac{\sin \pi \frac{k}{M^{j}}}{\pi \frac{k}{M^{j}}}\right)^{s} .
$$

In fact, after periodization for a big enough $j$ (such that $\left[-\frac{s / 2}{M^{j}}, \frac{s / 2}{M^{j}}\right] \in[-1 / 2,1 / 2]$ ) we have $\varphi_{j}^{s}(x)=$ $\varphi^{s}\left(M^{j} x\right)$ for $x \in[-1 / 2,1 / 2]$, i.e. $\varphi_{j}^{s}$ is a contracted B-spline $\varphi^{s}$.

Now, check that the periodic Strang-Fix conditions of order $s$ for the sequence $\left\{\varphi_{j}^{s}\right\}_{j}$ are valid and the corresponding sequence $\left\{b_{n}\right\}_{n}$ from (11) belongs to $\ell_{q}$ for any $q>1$. Indeed, let $n \neq 0$ and $r \in D\left(M^{j}\right)$, then

$$
\left|\widehat{\varphi_{j}^{s}}\left(M^{j} n+r\right)\right|=\left|\frac{\sin \pi \frac{M^{j} n+r}{M^{j}}}{\pi \frac{M^{j} n+r}{M^{j}}}\right|^{s}=\left|\frac{\sin \pi \frac{r}{M^{j}}}{\pi\left(n+\frac{r}{M^{j}}\right)}\right|^{s} \leq\left|\frac{\frac{r}{M^{j}}}{n+\frac{r}{M^{j}}}\right|^{s} \leq \frac{2^{s}}{(2|n|-1)^{s}}\left|\frac{r}{M^{j}}\right|^{s} .
$$

Also, we have that $b_{n}=\frac{2^{s}}{(2|n|-1)^{s}}, n \neq 0$, which implies that $\left\{b_{n}\right\}_{n} \in \ell_{q}$.

Next, for a given sequence $\left\{\widetilde{\varphi}_{j}\right\}_{j}$, we can consider linear combinations of splines in order to construct an appropriate sequence $\left\{\varphi_{j}\right\}_{j}$. For instance, in order to reduce noise contribution, it is reasonable to use smoothed version of samples instead of the exact samples of $f$ (see, e.g. 43]). Let $\tilde{\varphi}_{j}$ be such that

$$
\left\langle f, \widetilde{\varphi}_{j}\left(\cdot-M^{-j} k\right)\right\rangle=\frac{1}{2} f\left(M^{-j} k\right)+\frac{1}{4} f\left(M^{-j}(k+1)\right)+\frac{1}{4} f\left(M^{-j}(k-1)\right) .
$$

This means that $\widehat{\widetilde{\varphi}_{j}}(k)=\frac{1}{2}+\frac{1}{2} \cos 2 \pi \frac{k}{M^{j}}$. Thus, condition (10) is satisfied with $N=0$. Consider a periodized B-spline as a dual sequence $\left\{\varphi_{j}^{s}\right\}$, whose Fourier coefficients are defined by (51). Therefore,

$$
1-\widehat{\varphi_{j}}(k) \widehat{\varphi_{j}^{s}}(k)=1-\left(\frac{1}{2}+\frac{1}{2} \cos 2 \pi \frac{k}{M^{j}}\right)\left(\frac{\sin \pi \frac{k}{M^{j}}}{\pi \frac{k}{M^{j}}}\right)^{s} .
$$

Since, by Taylor's formula, $f_{1}(x):=1-\left(\frac{1}{2}+\frac{1}{2} \cos 2 \pi x\right)\left(\frac{\sin \pi x}{\pi x}\right)^{s}=\frac{1}{6}\left(6 \pi^{2}+s \pi^{2}\right) x^{2}+\mathcal{O}\left(x^{4}\right)$ as $x \rightarrow 0$, it is clear that the order of compatibility cannot be better than 2. Thus, using Lagrange's reminder for the case $s=2$, we can state that

$$
\left|f_{1}(x)\right| \leq \frac{|x|^{2}}{2 !} \max _{x \in[-1 / 2,1 / 2]}\left|f_{1}^{\prime \prime}(x)\right|=\frac{4}{3} \pi^{2}|x|^{2},
$$

which implies

$$
\left|1-\widehat{\varphi_{j}}(k) \widehat{\varphi_{j}^{s}}(k)\right| \leq \frac{4}{3} \pi^{2}\left|\frac{k}{M^{j}}\right|^{2}, \quad k \in D\left(M^{* j}\right) .
$$

Now, consider a linear combination of the shifted splines $\varphi_{j}^{s}$, which allows to provide a better order of compatibility. For instance, let $\varphi_{j}:=u_{1} \varphi_{j}^{s}+\frac{u_{2}}{2} \varphi_{j}^{s}\left(\cdot+\frac{1}{M^{j}}\right)+\frac{u_{2}}{2} \varphi_{j}^{s}\left(\cdot-\frac{1}{M^{j}}\right)$. The Fourier coefficients of $\varphi_{j}$ are

$$
\widehat{\varphi_{j}}(k)=\left(u_{1}\left(\frac{\sin \pi \frac{k}{M^{j}}}{\pi \frac{k}{M^{j}}}\right)^{s}+u_{2} \cos \left(2 \pi \frac{k}{M^{j}}\right)\left(\frac{\sin \pi \frac{k}{M^{j}}}{\pi \frac{k}{M^{j}}}\right)^{s}\right) .
$$

To find the compatibility order, we consider the function

$$
f_{2}(x):=1-\left(\frac{1}{2}+\frac{1}{2} \cos 2 \pi x\right)\left(\frac{\sin \pi x}{\pi x}\right)^{s}\left(u_{1}+u_{2} \cos (2 \pi x)\right) .
$$


From its Taylor's formula near the origin, we get

$$
f_{2}(x)=\left(1-u_{1}-u_{2}\right)+\frac{\pi^{2}}{6} x^{2}\left(u_{1} s+6 u_{1}+u_{2} s+18 u_{2}\right)+\mathcal{O}\left(x^{4}\right) .
$$

It is clear that for $s=4, u_{1}=11 / 6, u_{2}=-5 / 6$, the first two terms are vanished. In order to get constant value $b_{0}$ from condition (12), Moreover, using Taylor's reminder, we have

$$
\left|f_{2}(x)\right| \leq \frac{|x|^{4}}{4 !} \max _{x \in[-1 / 2,1 / 2]}\left|f_{2}^{(I V)}(x)\right|=\frac{32}{15} \pi^{4}|x|^{4} .
$$

This yields the weak compatibility of order 4 for $\left\{\varphi_{j}^{s}\right\}_{j}$ and $\left\{\widetilde{\varphi}_{j}\right\}_{j}$, i.e.,

$$
\left|1-\widehat{\widetilde{\varphi}_{j}}(k) \widehat{\varphi_{j}}(k)\right| \leq \frac{32}{15} \pi^{4}\left|\frac{k}{M^{j}}\right|^{4}, \quad k \in D\left(M^{* j}\right) .
$$

A similar procedure can be applied for the construction of a sequence $\left\{\varphi_{j}\right\}_{j}$, which is weakly compatible with a sequence of distributions $\left\{\widetilde{\varphi}_{j}\right\}_{j}$ corresponding to some differential operator. For instance, assume that

$$
\widehat{\varphi_{j}}(k)=1+c_{1}\left(2 \pi i M^{-j} k\right)^{2}
$$

and $\widehat{\varphi_{j}}(k)$ are defined by (52) with $s=4$. Let us try to achieve the weak compatibility of order 4 for the corresponding sequences $\left\{\varphi_{j}\right\}_{j}$ and $\left\{\widetilde{\varphi}_{j}\right\}_{j}$. For this, it is enough to apply Taylor's formula to the function

$$
f_{3}(x):=1-\left(1+c_{1}(2 \pi i x)^{2}\right)\left(\frac{\sin \pi x}{\pi x}\right)^{4}\left(u_{1}+u_{2} \cos (2 \pi x)\right)
$$

and chose $u_{1}=2 c_{1}+4 / 3$ and $u_{2}=1-u_{1}$ in (52). In particular, if $c_{1}=-1 / 4$, then

$$
\left|1-\widehat{\widetilde{\varphi}_{j}}(k) \widehat{\varphi_{j}}(k)\right| \leq \frac{7}{15} \pi^{4}\left|\frac{k}{M^{j}}\right|^{4}, \quad k \in D\left(M^{* j}\right),
$$

which implies the required fact.

\section{References}

[1] C. Bardaro, P. L. Butzer, R. L. Stens, G. Vinti, Approximation error of the Whittaker cardinal series in terms of an averaged modulus of smoothness covering discontinuous signals, Math. Anal. Appl. 316 (2006), no. 1, 269-306.

[2] C. Bardaro, P. L. Butzer, R. L. Stens, G. Vinti, Prediction by samples from the past with error estimates covering discontinuous signals, IEEE Trans. Inform. Theory $\mathbf{5 6}$ (2010), no. 1, 614633.

[3] R. Bergmann, J. Prestin, Multivariate anisotropic interpolation on the torus in: Approximation Theory XIV: San Antonio 2013, Springer Proceedings in Mathematics \& Statistics 83, Springer International Publishing Switzerland 2014, 27-44.

[4] C. De Boor, R. DeVore, A. Ron, Approximation from shift-invariant subspaces of $L_{2}\left(\mathbb{R}^{d}\right)$, Trans. Amer. Math. Soc. 341 (1994), no. 2, 787-806.

[5] C. De Boor, R. DeVore, A. Ron, Approximation orders of FSI spaces in $L_{2}\left(\mathbb{R}^{d}\right)$, Constr. Approx. 14 (1998), no. 3, 411-427. 
[6] J. L. Brown, JR., On the error in reconstructing a non-bandlimited function by means of the bandpass sampling theorem, J. Math. Anal. Appl. 18 (1967), 75-84.

[7] G. Brumme, Error estimates for periodic interpolation by translates, Wavelets, Images, and Surface Fitting, in: P.J. Laurent, A. le Méhauté, L.L. Schumaker (Eds.), Wavelets, Images and Surface Fitting, AK Peters Ltd., Boston, 1994, pp. 75-82.

[8] P. L. Butzer, A survey of the Whittaker-Shannon sampling theorem and some of its extensions, J. Math. Res. Expositio 3 (1983), 185-212.

[9] P. L. Butzer, J. R. Higgins, R. L. Stens, Classical and approximate sampling theorems: studies in the $L_{p}(\mathbb{R})$ and the uniform norm, J. Approx. Theory 137 (2005), no. 2, 250-263.

[10] D. Costarelli, G. Vinti, Approximation by nonlinear multivariate sampling Kantorovich type operators and applications to image processing, Numer. Funct. Anal. Optim. 34 (2013) 819-844.

[11] D. Costarelli, G. Vinti, Rate of approximation for multivariate sampling Kantorovich operators on some functions spaces, J. Int. Eq. Appl. 26 (2014), no. 4, 455-481.

[12] D. Costarelli and G. Vinti, Order of approximation for sampling Kantorovich operators, J. Int. Eq. Appl. 26 (2014), no. 3, 345-368.

[13] F.J. Delvos, Periodic interpolation on uniform meshes, J. Approx. Theory 51 (1987) 71-80.

[14] G. B. Folland, Fourier Analysis and Its Applications. American Mathematical Society. 1992.

[15] H. G. Feichtinger, N. Kaiblinger, Quasi-interpolation in the Fourier algebra, J. Approx. Theory 144 (2007), no. 1, 103-118.

[16] L. Grafakos, Classical Fourier analysis. Second edition. Springer, New York, 2008.

[17] K. Jetter, D. X. Zhou, Order of linear approximation from shift invariant spaces, Constr. Approx. 11 (1995), no. 4, 423-438.

[18] R.-Q. JIA, Refinable shift-invariant spaces: from splines to wavelets, in: C.K. Chui, L.L. Schumaker (Eds.), Approximation Theory VIII, vol. 2 (College Station, TX, 1995), Ser. Approx. Decompos., vol. 6, World Scientific Publishing, River Edge, NJ, 1995, pp. 179-208.

[19] R.-Q. Jia, Convergence rates of cascade algorithms, Proc. Amer. Math. Soc. 131 (2003), 17391749 .

[20] R.-Q. JiA, Approximation by quasi-projection operators in Besov spaces, J. Approx. Theory 162 (2010), no. 1, 186-200.

[21] A. Krivoshein, M. Skopina, V. Protasov, Multivariate Wavelet Frames. Industrial and Applied Mathematics, Springer. Singapore, 2016.

[22] A. Krivoshein, M. Skopina, Multivariate sampling-type approximation, Anal. Appl. 15 (2017), no. $4,521-542$.

[23] A. Krivoshein, M. Skopina, Approximation by frame-like wavelet systems, Appl. Comput. Harmon. Anal. 31 (2011), no. 3, 410-428.

[24] Yu. Kolomoitsev, A. Krivoshein, M. Skopina, Differential and falsified sampling expansions, J. Fourier Anal. Appl. 24 (2018), no. 5, 1276-1305. 
[25] Yu. Kolomoitsev, M. Skopina, Approximation by multivariate Kantorovich-Kotelnikov operators, J. Math. Anal. Appl. 456 (2017), no. 1, 195-213.

[26] E. Liflyand, Functions of bounded variation and their Fourier transforms. Applied and Numerical Harmonic Analysis. Birkhuser/Springer, Cham, 2019.

[27] E. Liflyand, S. Samko, R. Trigub, The Wiener algebra of absolutely convergent Fourier integrals: an overview, Anal. Math. Phys. 2 (2012), 1-68.

[28] F. Locher, Interpolation on uniform meshes by the translates of one function and related attenuation factors, Math. Comp. 37 (1981) 403-416.

[29] D. S. Lubinsky, A. Mate, P. Nevai, Quadrature sums involving $p$ th powers of polynomials, SIAM J. Math. Anal. 18 (1987), 53-544.

[30] O. Orlova, G. TAmberG, On approximation properties of generalized Kantorovich-type sampling operators, J. Approx. Theory 201 (2016), 73-86.

[31] G. PÖPlau, Multivariate periodische Interpolation durch Translate und deren Anwendung, Dissertation, Universität Rostock, 1995.

[32] G. Pöplau, F. Sprengel, Some error estimates for periodic interpolation on full and sparse grids, in: A. le Méhauté, C. Rabut, L.L. Schumaker (Eds.), Curves and Surfaces with Applications in CAGD, Vanderbilt University Press, 1997, pp. 355-362.

[33] H. J. Schmeisser, H. Triebel, Topics in Fourier Analysis and Function Spaces. Wiley. 1987.

[34] W. Sickel, F. Sprengel, Some error estimates for periodic interpolation of functions from Besov spaces, Proc. Conf. Multivariate Approx. Theory, Witten-Bommerholz, 1998, Birkhäuser, Oper. Theory, Adv. Appl. 110 (1999), 295-321.

[35] F. Sprengel, A class of periodic function spaces and interpolation on sparse grids, Numer. Funct. Anal. Optim. 21 (2000), no 1-2, 273-293.

[36] W. Sickel, Some remarks on trigonometric interpolation on the $n$-torus, Z. Anal. Anwend. 10 (1991), no. 4, 551-562.

[37] W. Sickel, Spline representations of functions in Besov-Triebel-Lizorkin spaces on $\mathbb{R}^{n}$, Forum Math. 2 (1990), no. 5, 451-475.

[38] M. Skopina, Band-limited scaling and wavelet expansions, Appl. Comput. Harmon. Anal. 36 (2014), 143-157.

[39] A. F. Timan, Theory of Approximation of Functions of a Real Variable, Pergamon Press, Oxford, London, New York, Paris, 1963.

[40] M. Unser, Sampling - 50 years after Shannon, Proceedings of the IEEE 88 (2000), 569-587.

[41] G. Vinti, L. Zampogni, Approximation results for a general class of Kantorovich type operators, Adv. Nonlinear Stud. 14 (2014), no. 4, 991-1011.

[42] Al. Zayed, Advances in Shannon's Sampling Theory. CRC Press, Boca Raton, FL, 1993.

[43] Q. Zhang, L. Wang, W. Sun, Signal denoising with average sampling, Digital Signal Processing 22 (2012), no. 2, 226-232.

[44] A. Zygmund, Trigonometric Series, Cambridge University Press, 1968. 